\title{
Pyocyanin, a Virulence Factor Produced by Pseudomonas aeruginosa, Alters Root Development Through Reactive Oxygen Species and Ethylene Signaling in Arabidopsis
}

\author{
Randy Ortiz-Castro, Ramón Pelagio-Flores, Alfonso Méndez-Bravo, León Francisco Ruiz-Herrera, \\ Jesús Campos-García, and José López-Bucio \\ Instituto de Investigaciones Químico-Biológicas, Universidad Michoacana de San Nicolás de Hidalgo. Edificio B3, Ciudad \\ Universitaria, C. P. 58030, Morelia, Michoacán, México
}

Submitted 8 August 2013. Accepted 31 October 2013.

Pyocyanin acts as a virulence factor in Pseudomonas aeruginosa, a plant and animal pathogen. In this study, we evaluated the effect of pyocyanin on growth and development of Arabidopsis seedlings. Root inoculation with $P$. aeruginosa PAO1 strain inhibited primary root growth in wild-type (WT) Arabidopsis seedlings. In contrast, single lasI- and double rhlI-/lasI- mutants of $P$. aeruginosa defective in pyocyanin production showed decreased root growth inhibition concomitant with an increased phytostimulation. Treatment with pyocyanin modulates root system architecture, inhibiting primary root growth and promoting lateral root and root hair formation without affecting meristem viability or causing cell death. These effects correlated with altered proportions of hydrogen peroxide and superoxide in root tips and with an inhibition of cell division and elongation. Mutant analyses showed that pyocyanin modulation of root growth was likely independent of auxin, cytokinin, and abscisic acid but required ethylene signaling because the Arabidopsis etr1-1, ein2-1, and ein3-1 ethylene-related mutants were less sensitive to pyocyanin-induced root stoppage and reactive oxygen species (ROS) distribution. Our findings suggest that pyocyanin is an important factor modulating the interplay between ROS production and root system architecture by an ethylene-dependent signaling.

The ecophysiology of plants cannot be understood without the microbial populations that proliferate outside and inside roots. Rhizobacterial species may impact root physiology through production of plant hormones such as auxin or cytokinins, by stimulating root growth, or by altering root system architecture. Moreover, many bacterial species provide protection against pathogens, tolerance to abiotic stress, and resistance to insect or herbivore attack, and even allelopathy may be due to root-associated microorganisms (Friesen et al. 2011; Ortiz-Castro et al. 2009). Bacteria communicate with plants through secreted signaling factors. These are small, diffusible

Corresponding author: J. López-Bucio; E-mail: jbucio@umich.mx; Telephone and Fax: +1 (52) 4433265788.

* The $\boldsymbol{e}$-Xtra logo stands for "electronic extra" and indicates that three supplementary figures are published online and that Figures 1, 2, 3, 6, 7, 8, 9 , and 10 appear in color online.

(C) 2014 The American Phytopathological Society molecules that are specifically released and then recognized by eukaryotic tissues. By producing different classes of signals, the bacteria can be recognized as pathogens or symbionts leading to very different host responses (Ortiz-Castro and LópezBucio 2013; Ortiz-Castro et al. 2011).

The Pseudomonas genus comprises ubiquitous gram-negative bacteria distributed in different environments and contains pathogenic species for plants (i.e., Pseudomonas syringae and $P$. aeruginosa). Other species have the ability to colonize the rhizosphere (i.e., P. aeruginosa, $P$. fluorescens, $P$. putida, $P$. aureofaciens, and $P$. chloraphilis), where they can act as plant-beneficial bacteria by antagonizing pathogens or through production of compounds that influence plant-disease resistance and growth (Venturi 2006). The ability of $P$. fluorescens $\mathrm{CHA0}$ and $P$. aeruginosa 7NSK2 to induce resistance in grapevine against Botrytis cinerea was recently demonstrated. Both strains also triggered an oxidative burst and phytoalexin accumulation in grape cells and primed leaves for accelerated phytoalexin production upon challenge with $B$. cinerea (Verhagen et al. 2010). Redox-active pyocyanin (PCN) secreted by $P$. aeruginosa 7NSK2 triggers systemic resistance to Magnaporthe grisea but enhances Rhizoctonia solani susceptibility in rice (De Vleeschauwer et al. 2006). These results suggest that Pseudomonas-derived metabolites can induce reactive oxygen species (ROS), which act as a double-edged sword in the interaction of rice with the hemibiotroph $M$. grisea and the necrotroph $R$. solani.

Gram-negative bacteria produce and use $N$-acyl- $L$-homoserine lactones (AHL) for cell-to-cell communication through a regulatory mechanism known as quorum sensing (QS), which links perception of bacterial cell density to gene expression (Fuqua et al. 1994). QS coordinates many physiological processes such as symbiosis, production of virulence factors, resistance to oxidative stress, antibiotic resistance, motility, and biofilm formation (Miller and Bassler 2001). In P. aeruginosa, two main QS signals, $N$-(3-oxododecanoyl)- $L$-homoserine lactone (C12-AHL) and $N$-butyryl- $L$-homoserine lactone (C4-AHL), are synthesized by the AHL synthases encoded by the lasI and rhlI genes, respectively. At high bacterial density, the transcription factor LasR binds to C12-AHL; whereas RhlR, another transcriptional regulator, binds to $\mathrm{C} 4-\mathrm{AHL}$ to activate the transcription of virulence genes (Bosgelmez-Tinaz 2003; de Kievit and Iglewski 2000; Fuqua and Greenberg 2002; Rumbaugh et al. 2000).

Roots have developed the capacity to recognize bacterial QS signals and adjust growth and development in response to 
these metabolites (Mathesius et al. 2003; Ortiz-Castro et al. 2008; von Rad et al. 2008). Recently, genetic, chemical, and plant-growth data were presented showing that, in $P$. aeruginosa, the lasI QS system controls the production of three diketopiperazines (DKP)—namely, cyclo(L-Pro-L-Val), cyclo(LPro-L-Phe), and cyclo(L-Pro-L-Tyr) - that were involved in plant growth promotion by this bacterium. Analysis of all three bacterial DKP in Arabidopsis thaliana seedlings provided detailed information indicative of an auxin-like activity, based on their efficacy at modulating root architecture, activation of auxin-regulated gene expression, and response of auxinsignaling related mutants (Ortiz-Castro et al. 2011).

$P$. aeruginosa is most studied for its importance as a human and plant pathogen. Surprisingly, many studies have revealed extensive conservation in its virulence mechanisms to infect evolutionary divergent hosts. One of these conserved virulence factors is PCN. For example, PCN participates in the fast killing of Caenorhabditis elegans, Drosophila melanogaster, and Mus musculus, likely by producing ROS (Lau et al. 2003, 2004a and b; Mahajan-Miklos et al. 1999). PCN is synthesized from chorismate through a series of complex steps mediated by proteins encoded by two $p h z A B C D E F G$ operons, and by the $p h z H, p h z M$, and $p h z S$ genes, which modify precursors into the tricyclic compound (Mavrodi et al. 2001; Rada and Leto 2013). PCN synthesis is regulated by QS, as several reports indicate that mutations in the lasI-lasR and rhlI-rhlR QS systems result in the loss of PCN production (de Kievit and Iglewski 2000; Rumbaugh et al. 2000; Schaber et al. 2004; Siehnel et al. 2010). Moreover, PCN itself functions as a QS signal, as indicated by the fact that i) it demonstrates cell density-dependent accumulation, ii) it is a small diffusible molecule that is recognized by adjacent cells, and iii) it triggers a specific transcriptional response (Dietrich et al. 2006), further complicating our understanding of its mechanisms of action.

Despite in vitro studies demonstrating that PCN interferes with multiple cellular functions in animals, its importance during bacteria-plant interactions is uncertain. This is partially caused by the difficulty in defining the contribution of PCN among the numerous virulence factors produced by $P$. aeruginosa during infection. Currently, the response of plant cells or whole organs to $P$. aeruginosa-produced PCN is unknown, and whether this compound causes cell damage or regulates fundamental cellular processes in plants remains to be clarified. To gain insight into how PCN might be functionally integrated into $P$. aeruginosa physiology during interaction with plants, the contribution of PCN to growth and development was assessed by comparing the Arabidopsis primary and lateral root responses to $P$. aeruginosa PAO1 and the QS-related mutants rhlI-, lasI-, and rhlI-/lasI- in direct interaction of the bacteria with the root system. We also provide detailed pharmacological evidence of PCN bioactivity on Arabidopsis seedlings and analyzed the growth of primary roots in response to PCN in wild-type (WT)-, auxin-, cytokinin-, ethylene-, and abscisic-acid-related Arabidopsis mutants. Our data conclusively indicate that PCN acts as a signaling molecule for root development likely affecting ROS production and ethylene signaling.

\section{RESULTS}

\section{AHL-mediated QS plays a role in growth and development} of Arabidopsis modulated by $P$. aeruginosa.

We first tested whether direct colonization of the Arabidopsis root with $P$. aeruginosa PAO1 and QS-related single rhlIand lasI-, and double rhlI-/lasI- mutants could affect growth of seedlings. In several experiments and times of co-cultivation, $P$. aeruginosa PAO1 caused primary root growth inhibi- tion, and the shoot system was unable to grow (Fig. 1B, G, and L). These effects were similar to those caused by the $P$. aeruginosa rhlI- mutant, defective on the AHL synthase that produces C4-AHL (Fig. 1C, H, and M). In contrast, co-cultivation with the $P$. aeruginosa lasI- single mutant defective on 3-oxoC12-AHL synthesis or with the rhlI-/lasI- double mutant failed to cause inhibition of growth and dramatically increased root and shoot biomass production of seedlings at 3, 6, and 9 days of co-cultivation (Fig. 1D, I, and N; and E, J and O, respectively). These data indicate that C12-AHL-mediated QS controls the production of factors that repress primary root growth. Interestingly, an analysis of hydrogen peroxide $\left(\mathrm{H}_{2} \mathrm{O}_{2}\right)$ in root tips of Arabidopsis seedlings co-cultivated for 9 days with WT $P$. aeruginosa and AHL-related mutants revealed a decrease in $\mathrm{H}_{2} \mathrm{O}_{2}$ in roots co-cultivated with WT and rhlI mutants and an increased accumulation of $\mathrm{H}_{2} \mathrm{O}_{2}$ in both lasI- and rhlI-/lasI- mutants (Fig. 2A to E). This highly contrasting response indicates that diffusible factors released by WT P. aeruginosa modulates the levels of $\mathrm{H}_{2} \mathrm{O}_{2}$ and perhaps other ROS likely involved in root system adjustment.

\section{AHL-mediated QS regulates PCN production in $P$. aeruginosa.}

P. aeruginosa releases $\mathrm{PCN}$ as a main virulence factor (De Vleesschauwer et al. 2006). To determine whether QS-related single rhlI- and lasI- and double rhlI-/lasI- mutants of $P$. aeruginosa could be defective on the production of PCN, we determined production of this metabolite in bacterial cell cultures by spectrophotometric analyses. It was found that PCN levels drastically decreased in single rhlI- and lasI- and double rhlI-/lasI- mutants when compared with $P$. aeruginosa PAO1 (Fig. 3). These data show that AHL-modulated QS plays an important role in $\mathrm{PCN}$ production.

\section{PCN alters $A$. thaliana root system architecture.}

To determine whether Arabidopsis plants could sense PCN and investigate how this compound affects plant morphogenesis, we evaluated Arabidopsis root developmental responses to pharmacological application of PCN. With this aim, Arabidopsis seedlings were germinated and grown on $0.2 \times$ Murashige-Skoog (MS) agar medium supplemented with PCN concentrations from 0.6 to $40 \mu \mathrm{M}$ and primary root growth was measured 10 days after germination (d.a.g.). PCN treatments showed a dose-dependent inhibitory effect of primary root growth, with $10 \mu \mathrm{M}$ PCN causing a $70 \%$ reduction in primary root length (Fig. 4A; Supplementary Fig. S1). In contrast, an induction of lateral root formation was evident from 0.6 to $2.5 \mu \mathrm{M}$ PCN, while inhibitory effects were recorded at higher concentrations (Fig. 4B). A stimulatory effect of PCN in lateral root density (LRD) was also observed with a threefold increase from 2.5 to $20 \mu \mathrm{M}$ concentration of this compound when compared with solvent-treated seedlings (Fig. 4C).

\section{PCN alters root hair development.}

Root hairs are root epidermal cells that participate in nutrient and water uptake and increase the exploratory potential of the root system. To analyze whether PCN could alter root hair development, we performed experiments in which Arabidopsis WT (Col-0) seedlings were germinated and grown on the surface of agar plates containing different concentrations of PCN from 0.6 to $40 \mu \mathrm{M}$. Root hair parameters were analyzed at 7 d.a.g. on primary roots of solvent-treated or PCN-treated seedlings. To investigate the effects of PCN on root hair density, we measured the trichoblast length and root hair length on seedlings subjected to different concentrations of this compound. Trichoblasts are the hair-forming root epidermal cells that form cell files along the root surface. We found a dose- 
dependent decrease in trichoblast length in response to $\mathrm{PCN}$ treatment (Fig. 5A), which correlated with increased root hair number and root hair length (Fig. 5B and C). Root hair development was located closer to the primary root tip in plants grown in medium supplied with $40 \mu \mathrm{M}$ PCN (Supplementary Fig. S2), clearly indicating the progression of cell differentiation toward the root meristem region. These results suggest that PCN can be perceived by roots and alter root system architecture and root hair development.

\section{PCN alters cell division}

\section{without affecting cell viability or integrity.}

Previous reports demonstrated the toxicity of PCN in different organisms. However, the effects of PCN inhibiting primary root growth of Arabidopsis seedlings suggested that this compound could play an important role in cell division or elongation. To investigate the patters of cell division in response to PCN, we analyzed the expression of $C y c B 1$ :uidA, which is expressed only in cells in the G2/M phase of the cell cycle and is a marker of mitotic activity (Colón-Carmona et al. 1999), and pPRZ1:uidA, which marks only active meristems (Sieberer et al. 2003). The inhibition of primary root growth under PCN concentrations of $5 \mu \mathrm{M}$ or higher correlated with the reduction in the number of cells expressing $C y c B 1$ :uidA in the primary root meristem and $\beta$-glucuronidase (GUS) expression of pPRZ1:uidA transgenic seedlings (Fig. 6A to $\mathrm{H}$ ). We also analyzed the gene expression of the cell nuclei marker AtHistH2B: YFP (yellow fluorescent protein) by confocal laser-scanning microscopy in seedlings stained with propidium iodide (PI) to determine whether PCN could cause cell death or damage of root tissues. Visualization of AtHistH2B:YFP in the nuclei of cells 7 d.a.g. showed that PCN-treated roots were, indeed, viable. In these cells, PI was unable to penetrate, even at concentrations higher than $40 \mu \mathrm{M}$ (Fig. 6I to L). Quantification of meristem length and number of cells expressing $C y c B 1$ :uidA clearly documented the repressing effects of PCN on cell proliferation in primary roots (Fig. $6 \mathrm{M}$ and $\mathrm{N}$ ). These results suggest that PCN regulates cell division without affecting cell integrity or meristem viability.

\section{PCN did not activate auxin inducible gene expression in Arabidopsis roots.}

Auxin is an important phytohormone involved in the modulation of several development processes in the root system. To

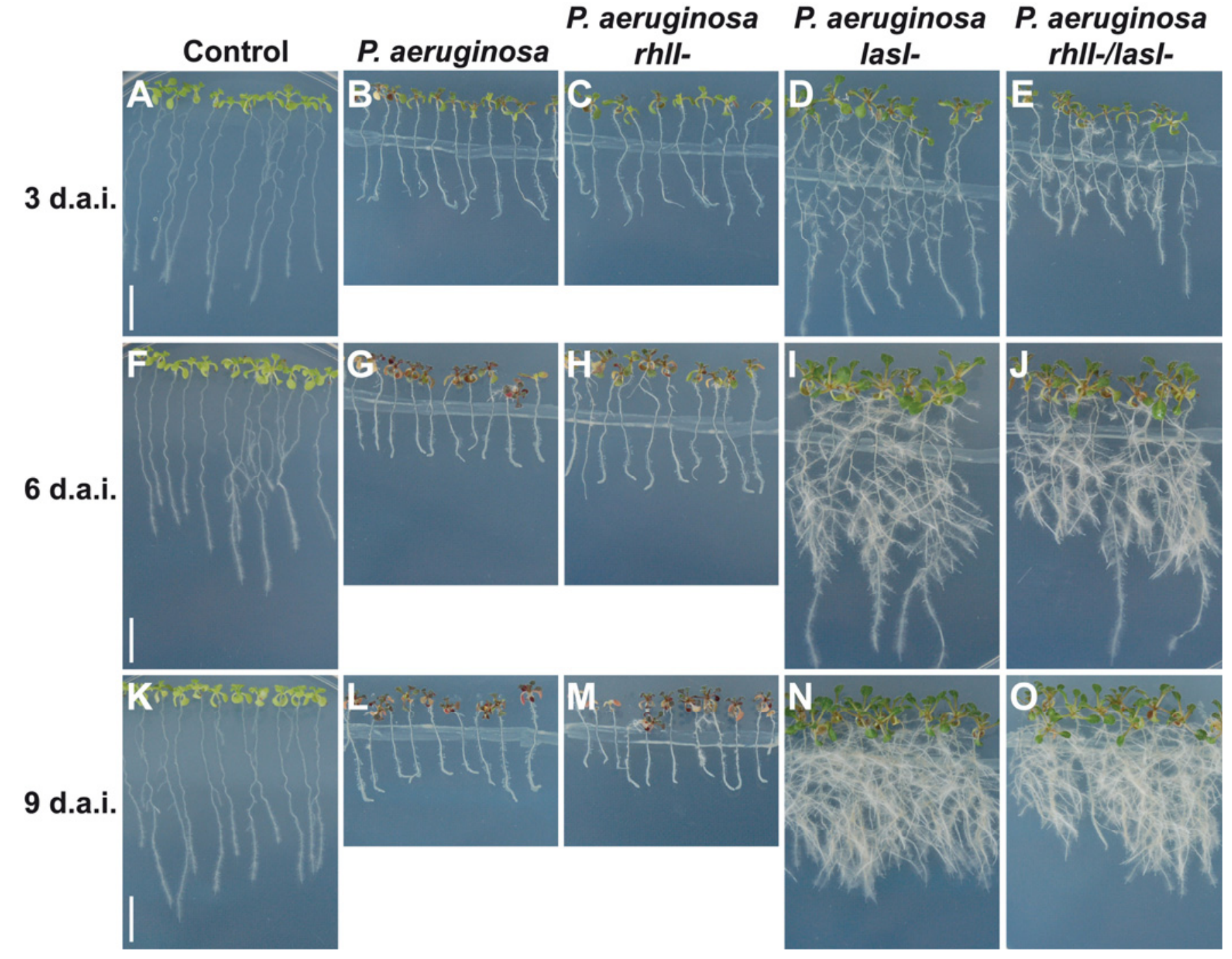

Fig. 1. Effect of co-cultivation with Pseudomonas aeruginosa wild-type (WT) and quorum-sensing (QS) mutant strains on plant growth. Six-day-old Arabidopsis thaliana seedlings were co-cultivated with WT P. aeruginosa or mutants defective on the AHL synthases LasI, RhlI, or RhlI/LasI at direct contact and grown for $\mathbf{A}$ to $\mathbf{E}, 3 ; \mathbf{F}$ to $\mathbf{J}, 6$; and $\mathbf{K}$ to $\mathbf{O}, 9$ days. Representative photographs were taken for plates from each treatment. This experiment was repeated three times with similar results. Notice the damage and inhibitory effect on root system architecture caused by the $P$. aeruginosa WT and rhlI- mutant and the strong induction on root system architecture and greening plants effect of the P. aeruginosa lasI- and rhlI-/lasI- mutants. Scale bar $=1 \mathrm{~cm}$. 
test whether PCN may or not function via auxin-regulated processes, we analyzed expression of DR5:uidA (Ulmasov et al. 1997) and BA3:uidA (Oono et al. 1998) auxin-inducible markers in transgenic Arabidopsis seedlings treated with PCN. Histochemical staining of roots of transgenic DR5:uidA and BA3:uidA seedlings that were grown for 10 days on $0.2 \times \mathrm{MS}$ medium supplemented with solvent, indole-3-acetic acid (IAA), or the indicated concentrations of PCN is shown in Figure 7. DR5:uidA expression in solvent-treated seedlings is located at the edges of the cotyledons and mainly at the root tip region (Fig. 7A and G). DR5:uidA seedlings grown in a concentration of $3 \mu \mathrm{M}$ IAA showed GUS activity throughout the shoot and primary root (Fig. 7B and $\mathrm{H}$ ), whereas BA3:uidA seedlings supplied with the same IAA concentration expressed GUS specifically at the root elongation region (Fig. $7 \mathrm{~N}$ and $\mathrm{T}$ ). When DR5:uidA and BA3:uidA seedlings were grown on PCN-supplied medium, the GUS expression remained similar in the shoot and primary root tip (Fig. 7C to F, I to L, O to R, and U to $\mathrm{X}$ ). These results suggest that $\mathrm{PCN}$ did not induce auxinresponsive gene expression in Arabidopsis seedlings.

PCN inhibits primary root growth of auxin-, cytokinin-, and abscisic-acid-related Arabidopsis mutants.

Several mutants with alterations in root development have been identified using screens for resistance to growth inhibitory amounts of phytohormones. Because PCN did not activate

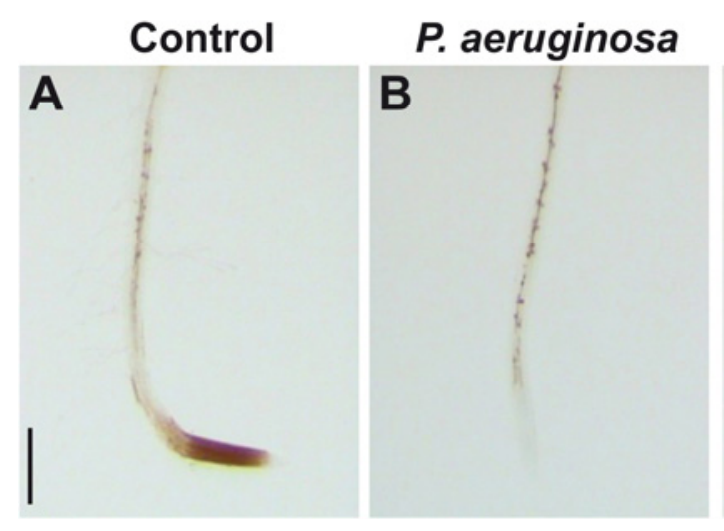

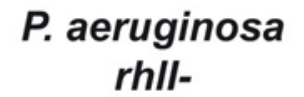

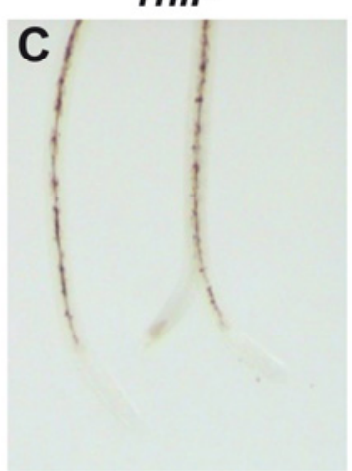

$P$. aeruginosa lasl-

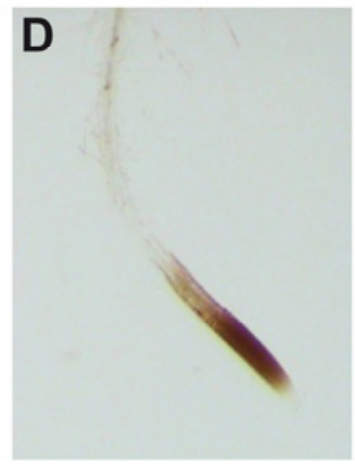

P. aeruginosa rhll-/lasl-

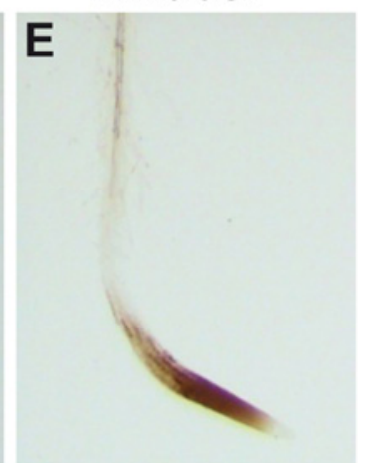

Fig. 2. Effect of co-cultivation of Pseudomonas aeruginosa wild-type (WT) and quorum-sensing (QS) mutant strains on $\mathrm{H}_{2} \mathrm{O}_{2}$ accumulation in the primary root meristem of Arabidopsis thaliana seedlings. Four-day-old A. thaliana seedlings were co-cultivated with WT P. aeruginosa or mutants defective on the AHL synthases RhlI, LasI, or RhlI/LasI at a distance of $5 \mathrm{~cm}$ from the primary root tip and grown for 8 days. Representative photographs of primary root of A, control seedlings or co-cultivated with $\mathbf{B}$, WT P. aeruginosa and $\mathbf{C}$, rhll; $\mathbf{D}$, lasI; or E, rhlI/lasI mutants. Arabidopsis seedlings were treated with a solution of 3,3'-diaminobenzidine (DAB). In the presence of $\mathrm{H}_{2} \mathrm{O}_{2}$, DAB polymerizes, forming a dark, red-brown coloration in plant tissues. This experiment was repeated three times with similar results. Scale bar $=500 \mu \mathrm{m}$.

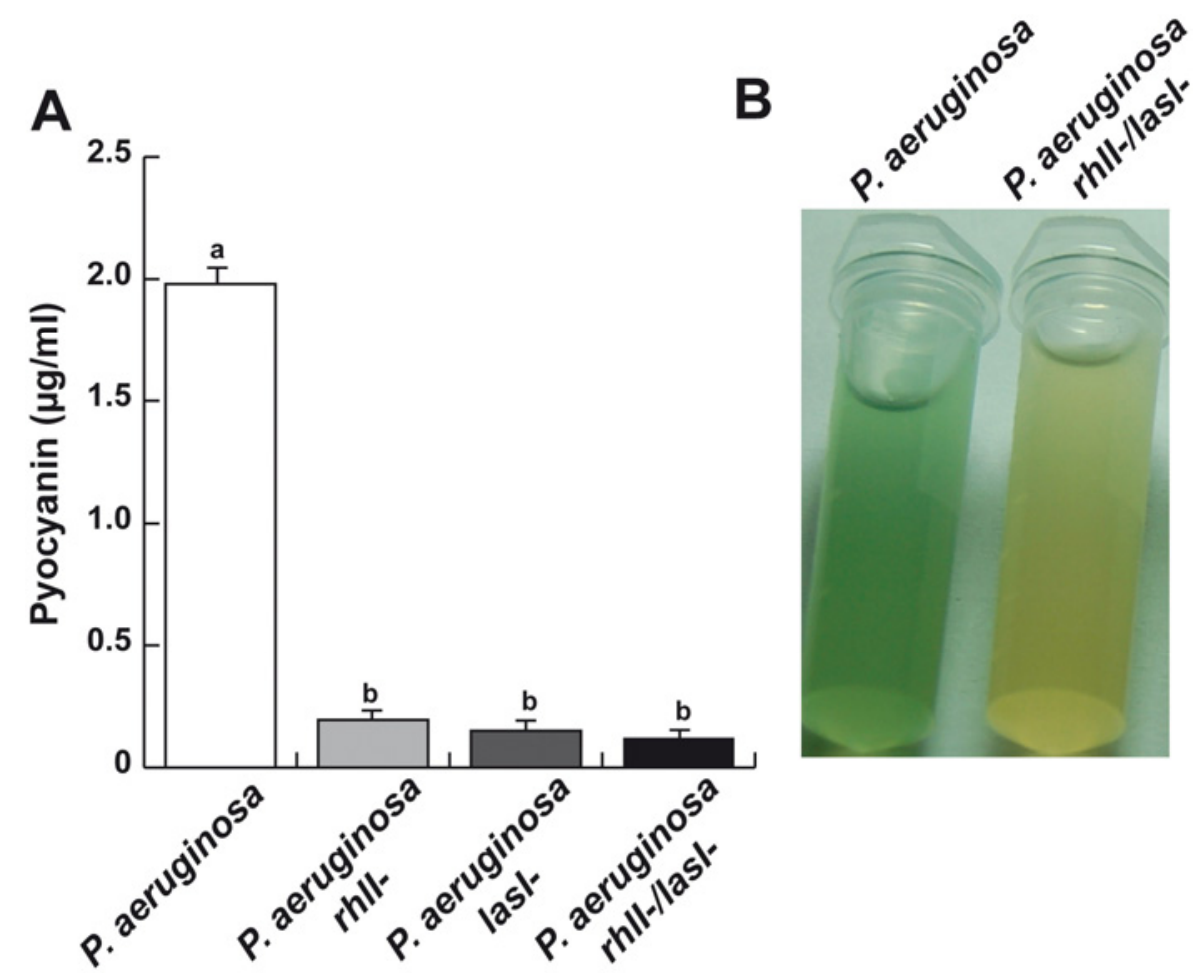

Fig. 3. Pyocyanin production in Pseudomonas aeruginosa wild-type (WT) and quorum-sensing (QS) mutant strains. A, Pyocyanin production: cells were grown in Luria-Bertani medium at $37^{\circ} \mathrm{C}$ for $48 \mathrm{~h}$, the supernatant fractions were separated, and the amount of pyocyanin $\left(\mu \mathrm{g} \mathrm{ml} l^{-1}\right)$ in each fraction was determined by the chloroform:acid extraction procedure. Values represent the mean of three independent experiments \pm standard deviation. B, Representative photograph of pyocyanin production as observed by the green color of culture grown in liquid medium. 

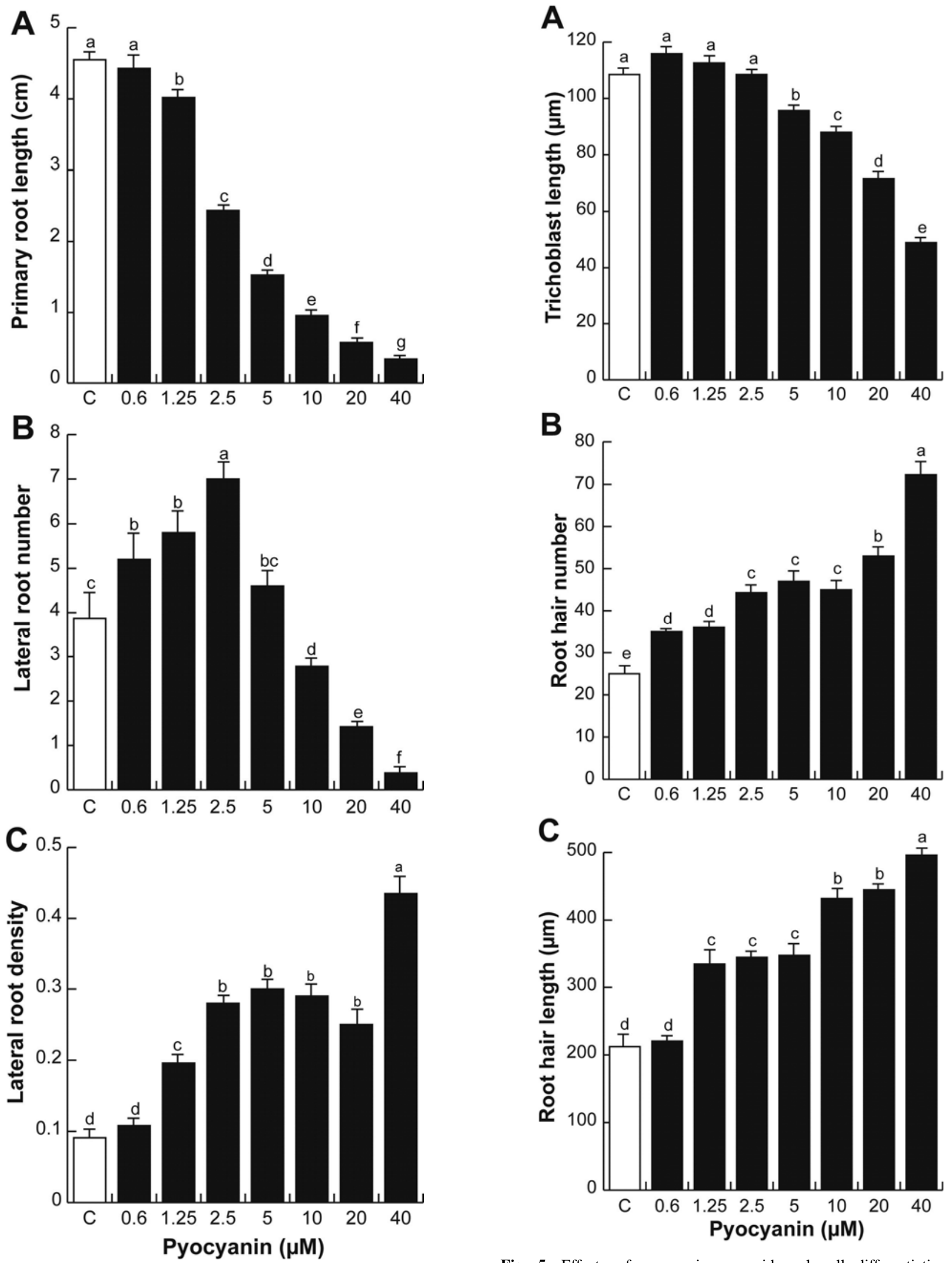

Fig. 4. Effect of pyocyanin on Arabidopsis root system architecture. Arabidopsis wild-type (WT) (Col-0) seedlings were germinated and grown for 10 days under increasing pyocyanin concentrations. A, Primary root length; B, lateral root number; C, lateral root density. Values shown represent the mean \pm standard deviation $(n=30)$. Different letters represent means statistically different at the 0.05 level. The experiment was repeated three times with similar results.

Fig. 5. Effects of pyocyanin on epidermal cell differentiation. A, Trichoblasts length; B, root hair number; C, root hair length. Arabidopsis thaliana seedlings were grown for 5 days on $0.2 \times$ Murashige-Skoog medium supplemented with the indicated concentrations of pyocyanin. Data points indicated mean \pm standard deviation $(n=20)$. Results show mean of 10 epidermal cells located in the root hair forming zone of the primary root. This experiment was repeated twice with similar results. Different letters indicate statistical differences at $P<0.05$. 

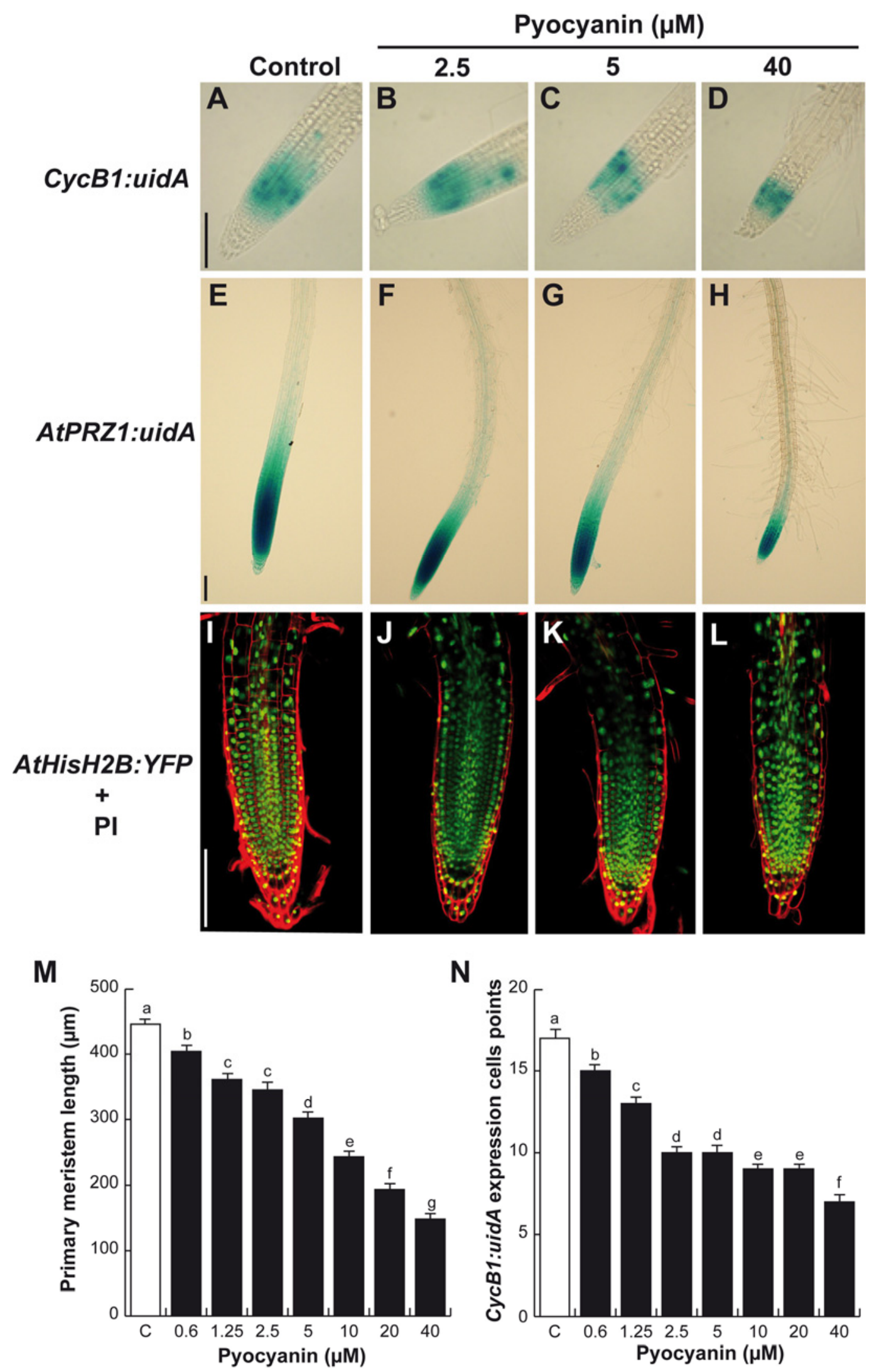

Fig. 6. Effect of pyocyanin on cell division and meristem viability. Arabidopsis thaliana seedlings expressing the CycB1:uidA, AtPRZ1:uidA, or AtHistH2B:YFP markers were grown for 5 days on $0.2 \times$ Murashige-Skoog medium supplemented with the indicated concentrations of pyocyanin. A to $\mathbf{H}$, Plants were stained for $\beta$-glucoronidase activity and cleared to show gene expression. I to L, Transgenic Arabidopsis seedlings expressing the AtHisH2B:YFP marker were stained with propidium iodide to determine cell structure and viability. Photographs show representative individuals from at least 20 stained plants. The experiment was replicated twice with similar results. Scale bar $=100 \mu \mathrm{m}$. M, The $C y c B 1:$ idA expression domain in response to pyocyanin was measured and $\mathbf{N}$, cells expressing this marker were counted. Data points represent the mean \pm standard deviation $(n=20)$. The experiment was replicated two times with similar results. Different letters indicate statistical differences at $P<0.05$. 
auxin-inducible markers, we decided to confirm whether PCN operates or not in a genetically defined auxin pathway. With this aim, Arabidopsis WT (Col-0) seedlings and auxin-related mutants tirlafb2afb3, arf7arf19, axr1-3, and auxl-7 were evaluated in primary root growth response assays to $10 \mu \mathrm{M}$ PCN. PCN treatment caused a $70 \%$ inhibition in primary root growth in WT plants compared with solvent-treated seedlings (Supplementary Fig. S3). When tirlafb2afb3, arf7arf19, axr 1-3, and aux 1-7 were grown in medium supplied with 10 $\mu \mathrm{M}$ PCN, the inhibition in primary root growth was similar to that observed in WT plants. The results of both auxinresponsive gene expression and the root response of auxinrelated mutants to PCN suggest that auxin is not involved in plant perception of PCN.

In addition, we evaluated the involvement of cytokinin, abscisic acid, and ethylene signaling in response to $\mathrm{PCN}$ by evaluating the primary root growth of Arabidopsis double mutants defective on cytokinin receptors (crel-12ahk2-2 and crel12ahk3-3), abscisic acid signaling (abil and abi3), and ethylene signaling (ein2-1 and ein3-1). The primary root growth of auxin, cytokinin, and abscisic acid mutants was normally inhibited by PCN, indicating that these phytohormones are unlikely mediating the cellular effects of PCN. Interestingly, an analysis of ethylene response mutants (ein2-1 and ein3-1) showed a small yet statistically significant resistance of primary root growth to inhibition by PCN, indicating that ethylene might be a signal that mediates the plant response to PCN.

\section{A role of ethylene signaling in root response to $\mathrm{PCN}$.}

To further define the particular role of ethylene signaling in the Arabidopsis developmental responses to PCN, we investigated the sensitivity of primary root responses to several PCN concentrations of Arabidopsis WT seedlings and etr1-1, ein2-1, and ein3-1 mutants. PCN was supplied to the growth medium in concentrations from 0.3 to $10 \mu \mathrm{M}$ and the primary root growth of all four lines was measured. Interestingly, we found than etrl-1 and ein2-1 showed resistance to inhibition of primary root growth compared with WT seedlings, while ein3-1 did not show clear resistance to PCN (Fig. 8A). To further determine the participation of ethylene signaling in the responses to $\mathrm{PCN}$, we used $\mathrm{AgNO}_{3}$, a well-known blocker of ethylene action. We found that, when Arabidopsis seedlings were grown on medium supplemented with $5 \mu \mathrm{M} \mathrm{PCN}$ and $5 \mu \mathrm{M} \mathrm{AgNO}_{3}$, the inhibitor reduced the effect of PCN in both primary root growth and root hair development (Fig. 8B and $\mathrm{C}$ to $\mathrm{J}$ ). This restoration of primary root growth in plants grown on medium supplemented with $\mathrm{PCN}$ and $\mathrm{AgNO}_{3}$ correlated with normalization of root hair differentiation process caused by PCN (Fig. $8 \mathrm{G}$ to $\mathrm{J}$ ). These results suggest that ethylene signaling plays a role in root architectural responses to $\mathrm{PCN}$.

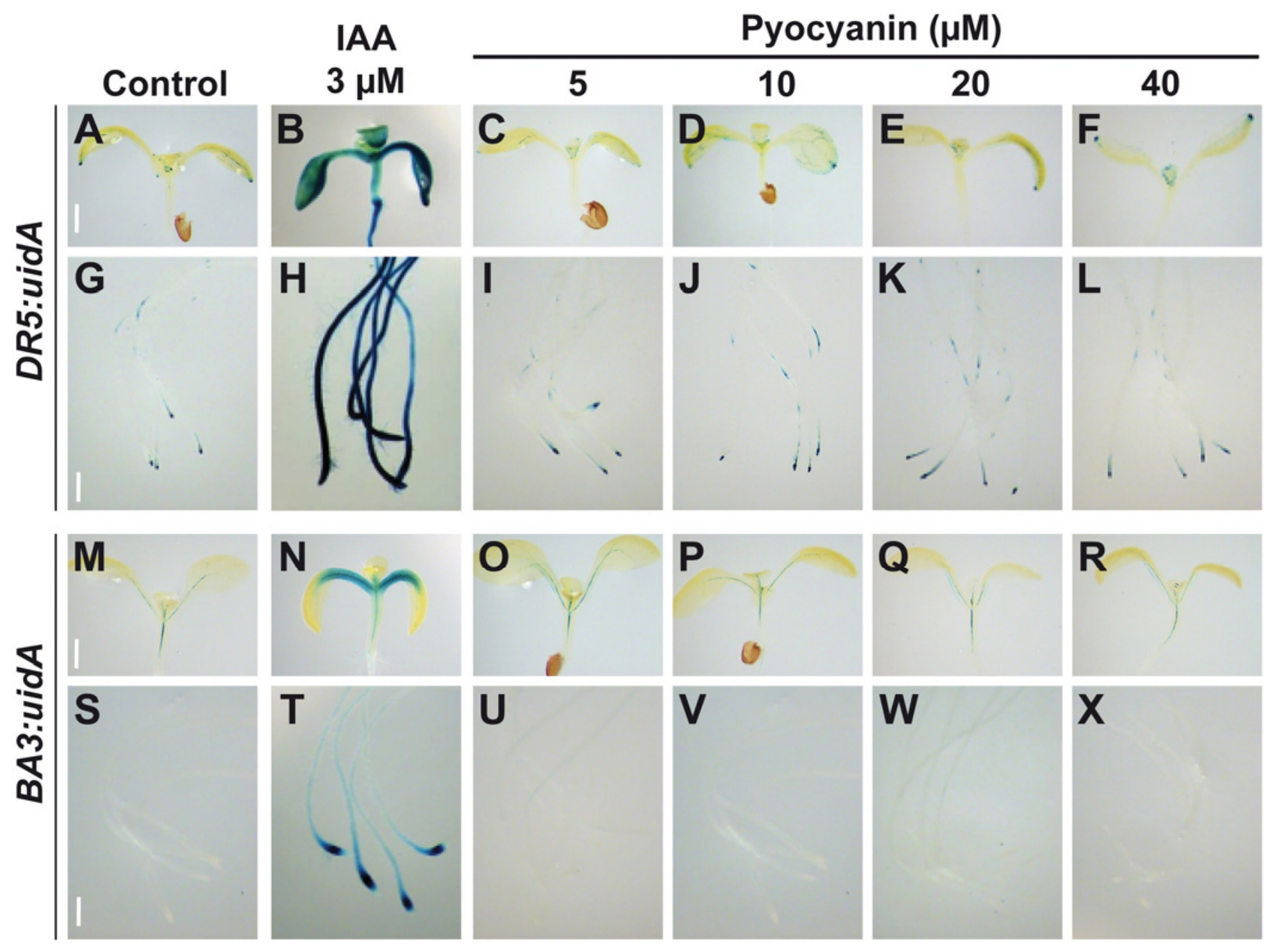

Fig. 7. Effect of pyocyanin on auxin-regulated gene expression. $\mathbf{A}$ to $\mathbf{L}$, DR5: uidA and $\mathbf{M}$ to $\mathbf{X}$, BA3: uidA gene expression in transgenic seedlings grown on $0.2 \times$ Murashige-Skoog (MS) agar medium for 6 days and then transferred into 24 -well cell culture plates (10 seedlings per well) containing 2 ml of $0.2 \times$ MS liquid medium supplied with the indicated concentrations of indole-3-acetic acid (IAA) or pyocyanin and incubated for $10 \mathrm{~h}$. Seedlings were stained for $\beta$-glucuronidase activity and cleared for microscopy analysis. Photographs show representative individuals from at least 30 stained plants (scale bars $=500 \mu \mathrm{m})$. 

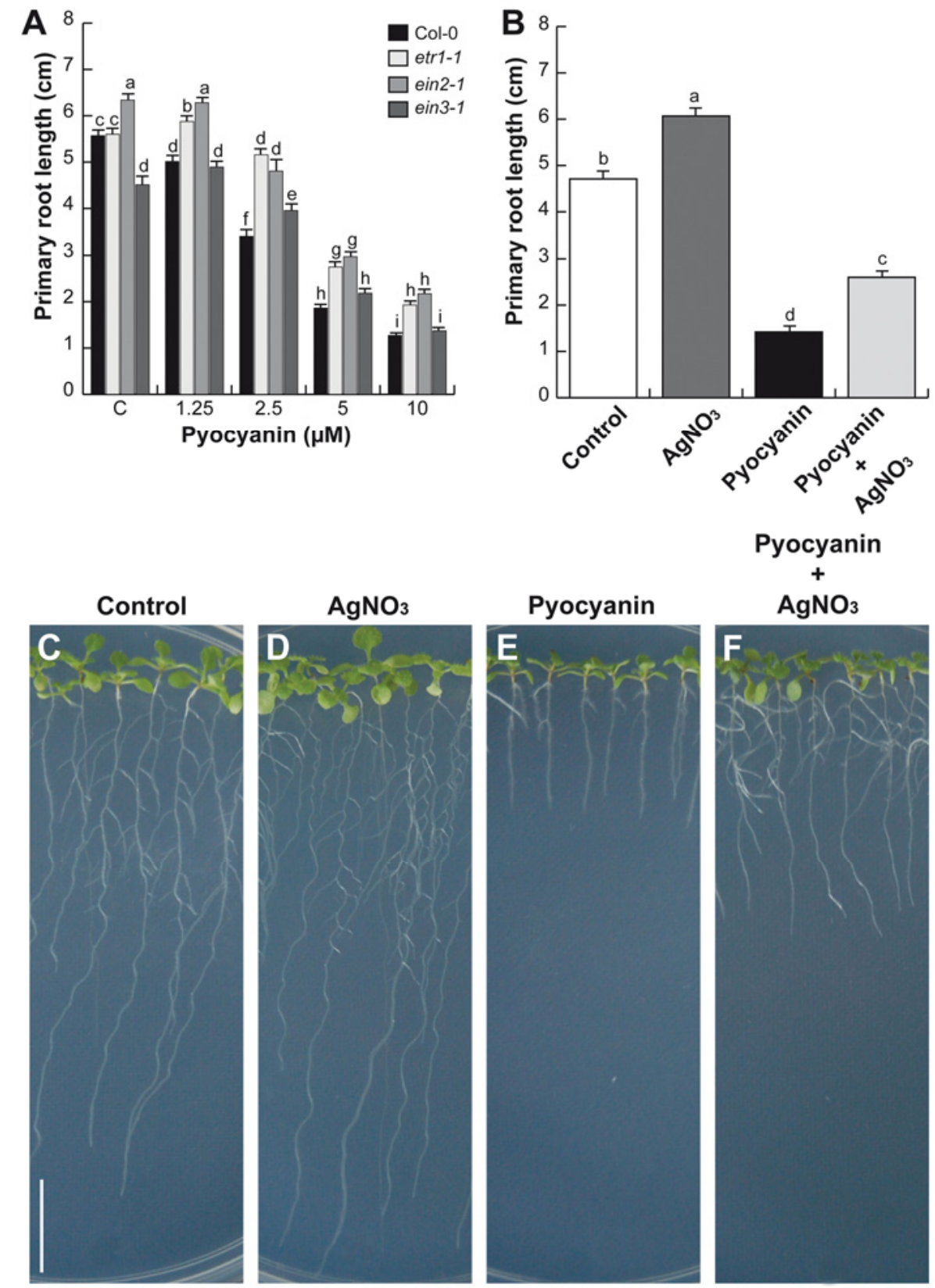

Pyocyanin
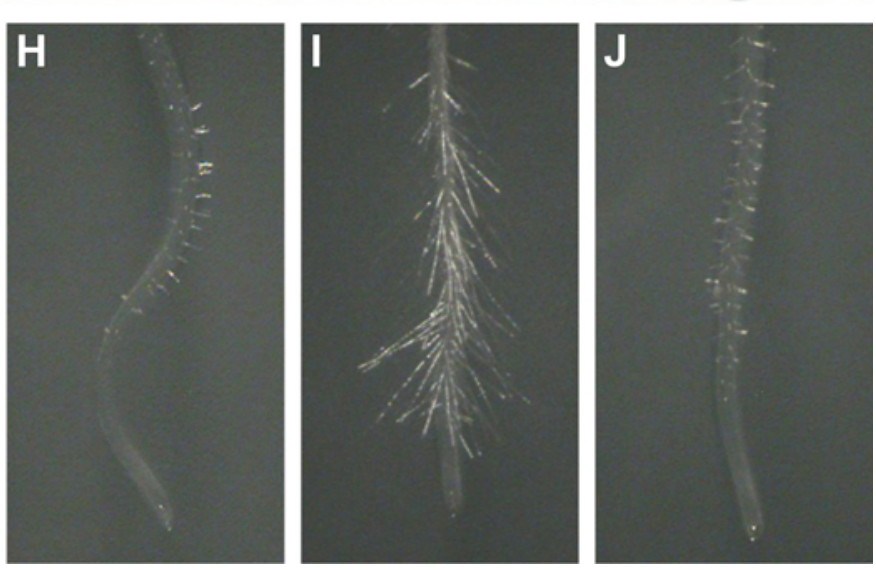

Fig. 8. Role of ethylene signaling in pyocyanin-induced primary root inhibition of Arabidopsis seedlings. A, Arabidopsis thaliana wild-type (WT) and etr11, ein2-1, and ein3-1 ethylene mutant seedlings were grown for 12 days on $0.2 \times$ Murashige-Skoog (MS) medium supplemented with the indicated concentrations of pyocyanin. B, Arabidopsis WT (Col-0) seedlings were grown for 12 days on $0.2 \times$ MS agar medium supplemented with $5 \mu \mathrm{M}$ pyocyanin and $\mathrm{AgNO}_{3}$. Data points show the mean \pm standard deviation $(n=30)$. Representative photographs of $\mathbf{C}$ to $\mathbf{F}$, Arabidopsis root system architecture and $\mathbf{G}$ to $\mathbf{J}$, root hair development under the different treatments are shown. Different letters indicate means that differ statistically at $P<0.05$. The experiment was repeated three times with similar results. 


\section{PCN induces ROS production dependent of ethylene signaling.}

An important toxicity mechanism by which PCN damages eukaryotic hosts is the production of ROS (Liu and Nizet 2009). To test whether the effects of PCN on primary root growth were accompanied by an induction of ROS, we analyzed ROS accumulation in primary root tips by confocal microscopy using fluorochromes to detect total ROS and superoxide $\left(\mathrm{O}^{2-}\right)$. To test the role of ethylene signaling in ROS induction by PCN, we grew Arabidopsis seedlings on $0.2 \times \mathrm{MS}$ medium supplemented with or without $5 \mu \mathrm{M} \mathrm{PCN}, \mathrm{AgNO}_{3}$, or PCN plus $\mathrm{AgNO}_{3}$ and, 7 d.a.g., the seedlings were incubated with $2^{\prime}, 7^{\prime}$-dichlorofluorescein diacetate (H2DCF-DA) or dihydroethidium (DHE) to detect total ROS and $\mathrm{O}^{2-}$ in the primary root tip by confocal microscopy. As expected, we found that total $\mathrm{ROS}$ and $\mathrm{O}^{2-}$ increased in plants treated with PCN (Fig. 9C and $\mathrm{H}$ ). This increase in ROS was similar to that induced by paraquat, a generator of ROS commonly used to evaluate ROS production in different systems (Fig. 9E and J). Interestingly, when plants are supplied with the ethylene perception blocker $\mathrm{AgNO}_{3}$, the levels of $\mathrm{ROS}$ and $\mathrm{O}^{2-}$ were reduced in control seedlings (Fig. 9B and G) or in seedlings supplied with PCN (Fig. 9D and I). Quantification of fluorescence confirms that PCN provokes an ROS accumulation which is dependent of ethylene signaling (Fig. 9K and L).

In another series of experiments, we analyzed the levels of $\mathrm{H}_{2} \mathrm{O}_{2}$ in ethylene-related mutants treated with different concentrations of PCN, whose levels clearly changed in Arabidopsis root tips (Fig. 10). We found that PCN reduces the levels of $\mathrm{H}_{2} \mathrm{O}_{2}$ on primary root tips in a dose-dependent way (Fig. 10A to F). However, in etr1-1, ein2-1, and ein3-1 seedlings, the levels of $\mathrm{H}_{2} \mathrm{O}_{2}$ were sustained even at concentrations of 1.25 and $2.5 \mu \mathrm{M}$ PCN that drastically affect root growth (Fig. 10G to $\mathrm{X})$. This sustained production of $\mathrm{H}_{2} \mathrm{O}_{2}$ indicates that the $\mathrm{PCN}$ mechanism of signaling involves the ethylene pathway and that it is probably related to the resistance of primary root growth when the plants are grown on PCN.

\section{DISCUSSION}

Plant roots are colonized by an immense number of microbes, referred to as the root microbiome. Selected strains of beneficial soilborne bacteria can protect against abiotic stress and prime the plant immune system against a broad range of pathogens. Pseudomonas spp. rhizobacteria represent one of the most abundant genera of the root microbiome. Rhizobacteria can influence root architecture; most prominently, by enhancing lateral root formation and root hair development. This can be done by producing phytohormones or bacterial QS signals that are perceived at the root tip to adjust cell proliferation and growth.

Our previous work has shown that co-cultivation of Arabidopsis seedlings with $P$. aeruginosa inhibits primary root growth, which is determined by the rate of cell division in the meristematic zone and the extent of cell expansion in the elongation zone. This leads to an acceleration of lateral root growth as a result of increased rates of cell division in the pericycle (Ortiz-Castro et al. 2011). Interestingly, co-cultivation of Arabidopsis with the QS-related mutants rhlI-, lasI-, and rhlI-/lasIcaused a decreased inhibition of root growth and a concomitant phytostimulation (Fig. 1), which can be likely explained by either a decreased production of virulence factors or stimulation of root developmental processes as P. aeruginosa lasIand rhlI-/lasI- mutants overproduce cyclodipeptides with auxin activity (Ortiz-Castro et al. 2011). Most likely, the beneficial effects of co-cultivation with lasI- and rhlI-/lasI- bacterial strains may be due to a combination of both processes.
The beneficial effect of Pseudomonas spp. to plants by means of regulating root architecture was recently confirmed by Zamioudis and associates (2013). By employing a germfree experimental system, the authors showed the ability of selected Pseudomonas strains to promote plant growth and drive developmental plasticity in the roots of Arabidopsis by inhibiting primary root elongation and promoting lateral root and root hair formation. By studying cell-type-specific developmental markers and employing genetic and pharmacological approaches, it was demonstrated the crucial role of auxin signaling and transport in rhizobacteria-stimulated changes in the root system architecture of Arabidopsis. The authors further show that Pseudomonas spp.-elicited alterations in root morphology and that rhizobacteria-mediated systemic immunity are mediated by distinct signaling pathways.

Root growth depends on maintaining the proper balance between cell division and differentiation. In the primary root, cells originate from a stem cell center at the tip. Progeny of these stem cells rapidly divide in a transit-amplifying zone known as the meristem, after which they undergo massive increases in cell volume in the elongation zone. Once fully elongated, cells enter the maturation zone, in which they differentiate into various cell types.

One of the factors of virulence and survival of $P$. aeruginosa is the production of secondary metabolites (i.e., phenazines, which have antibiotic properties) including PCN (1-hydroxy5-methylphenazine), a blue-green pigment with redox properties (Lau et al. 2004a; Liu and Nizet 2009). PCN synthesis is regulated by the lasR and $r h l R$ QS systems (de Kievit and Iglewski 2000; Rumbaugh et al. 2000; Schaber et al. 2004; Siehnel et al. 2010) and, in agreement with these previous results, we found a decreased production of PCN in lasI and rhlI/lasI P. aeruginosa mutants (Fig. 3). Although all three QSrelated $P$. aeruginosa mutants tested have a significant defect in PCN production, the rhlI mutant inhibited growth and affected root architecture similarly to the WT Paol strain. This result indicates that loss of PCN production is not the only factor by which $P$. aeruginosa inhibits root growth or decreases $\mathrm{H}_{2} \mathrm{O}_{2}$ level in the root tip. A second factor which affects root growth and is present in rhlI mutants is C12-AHL. Our previous work demonstrated that C12-AHL but not C4- or C6-AHL is very active in inhibiting primary root growth (Ortiz-Castro et al. 2008), thus explaining why rhlI mutants still repress primary root growth.

PCN can easily penetrate biological membranes and directly accept electrons from reducing agents such as NADPH and reduced glutathione, then transfer the electrons to oxygen to generate ROS such as $\mathrm{H}_{2} \mathrm{O}_{2}$ and $\mathrm{O}^{2-}$ at the expenses of host antioxidant systems such as glutathione and catalase (O'Malley et al. 2004). Several reports have documented that PCN is an important virulence factor of $P$. aeruginosa. Its induction through quorum signaling correlated with the biofilm growth stage of the bacterium. Although PCN has a wide range of toxic effects in animal cells, the proposed basis of its toxicity is production of $\mathrm{O}^{2-}$ anions and downstream ROS by oxidizing NADPH (O'Malley et al. 2004; Lau et al. 2004a; Liu and Nizet 2009). To the best of our knowledge, there is a lack of information about the levels of PCN released by $P$. aeruginosa when colonizing plant roots. However, some reports, mainly from animal systems, have shown that PCN accumulates in low micromolar levels in $P$. aeruginosa host cells. Wilson and associates (1998) showed that PCN levels varied between 78.5 and $128.5 \mu \mathrm{M}$ in the sputum of patients with cystic fibrosis (CF). Hunter and associates (2012) analyzed the level of PCN of $47 \mathrm{CF}$ patients, identifying concentrations up to $48 \mu \mathrm{M}$ in patients with severe CF. In another study by Price-Whelan and associates (2007), the PCN concentration reached $100 \mu \mathrm{M}$ in 
the stationary phase. Dietrich and associates (2006) showed that PCN activates genes related to redox homeostasis, iron acquisition, and virulence by using DNA microarrays and quantitative reverse-transcriptase polymerase chain reaction. In this work, the authors reported that $P$. aeruginosa PAO1 and PA14 produce 10 and $55 \mu \mathrm{M}$ PCN, respectively, during the stationary phase, and demonstrated that PCN can act as signaling molecule at these concentrations.
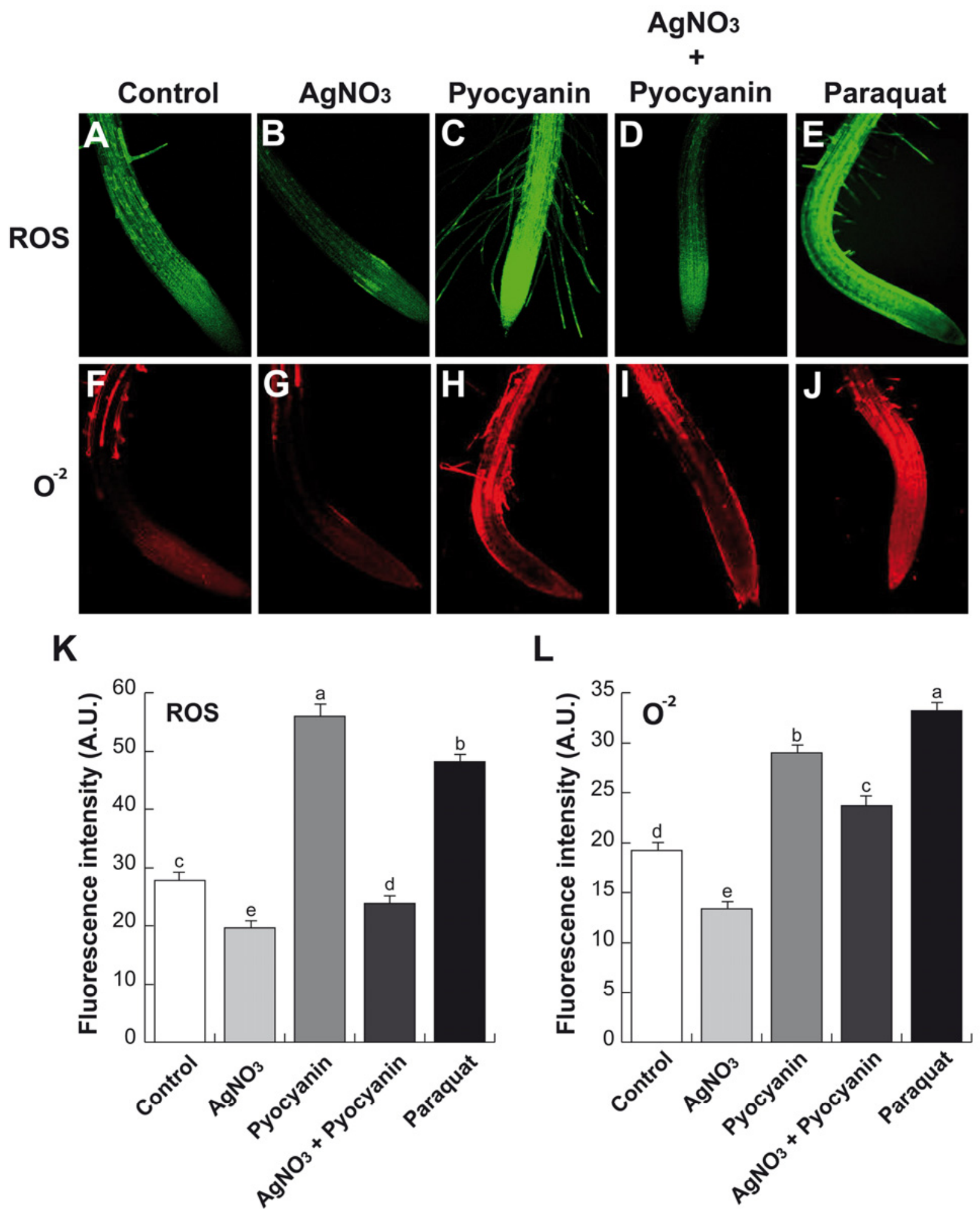

Fig. 9. Role of ethylene signaling in reactive oxygen species (ROS)-induced production by pyocyanin. Representative photographs of the detection of endogenous $\mathbf{A}$ to $\mathbf{E}, \mathrm{ROS}$ and $\mathbf{F}$ to $\mathbf{J}, \mathrm{O}^{2-}$ with $2^{\prime}, 7^{\prime}$-dichlorofluorescein diacetate (H2DCF-DA) and dihydroethidium (DHE), respectively, which were determined in primary roots of Arabidopsis seedlings grown for 7 days on $0.2 \times$ Murashige-Skoog agar medium supplemented with $5 \mu \mathrm{M}$ pyocyanin and $\mathrm{AgNO}_{3}$ or with $0.1 \mu \mathrm{M}$ paraquat. H2DCF-DA and DHE fluorescence signals from primary root tips $(n=10)$ for $\mathbf{K}$, ROS and $\mathbf{L}, \mathrm{O}^{2-}$ were quantified using the ImageJ program. The graph is expressed in arbitrary units. Values in $\mathrm{K}$ and $\mathrm{L}$ represent the mean \pm standard deviation $(n=30)$. Different letters are used to indicate means that differ statistically at $P<0.05$. The experiment was repeated three times with similar results. Photographs are representative individuals of at least 10 seedlings analyzed. Scale $b a r=100 \mu \mathrm{m}$. 


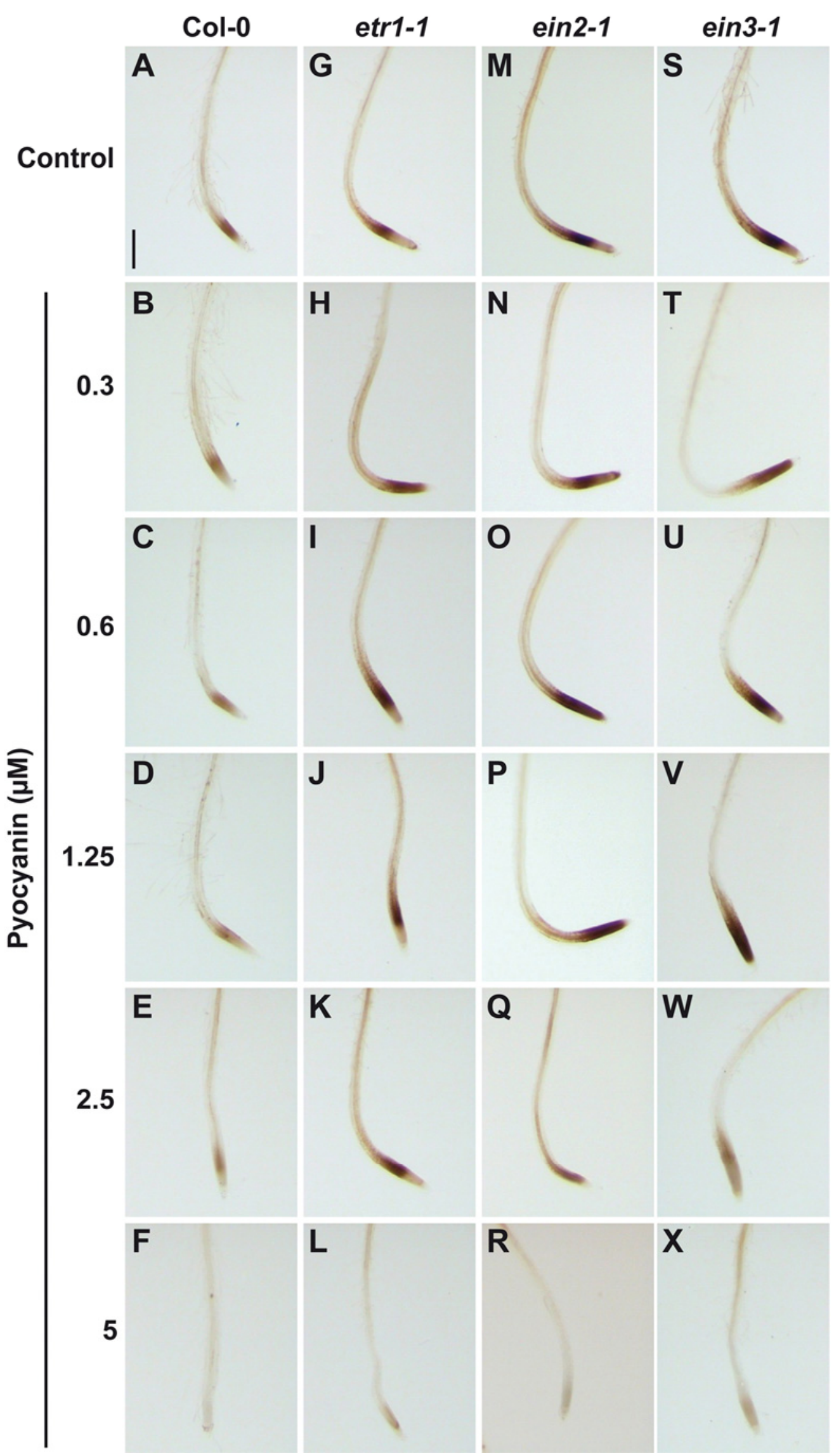

Fig. 10. Effect of pyocyanin on $\mathrm{H}_{2} \mathrm{O}_{2}$ accumulation in the primary root meristem of Arabidopsis thaliana wild-type (WT) (Col-0) and ethylene-related mutants etr1, ein2, and ein3. Histochemical detection of $\mathrm{H}_{2} \mathrm{O}_{2}$ with 3,3'-diaminobenzidine staining in $\mathbf{A}$ to $\mathbf{F}$, Arabidopsis thaliana WT (Col-0) and $\mathbf{G}$ to $\mathbf{L}$, etr1-1; $\mathbf{M}$ to $\mathbf{R}$, ein2-1; and $\mathbf{S}$ to $\mathbf{X}$, ein3-1. Arabidopsis seedlings were grown for 7 days on $0.2 \times$ Murashige-Skoog medium supplemented with the indicated concentrations of pyocyanin. Photographs show representative individuals from at least 30 stained plants. Scale bar $=500 \mu \mathrm{m}$. 
Based on the finding that PCN is capable of killing fungi and is toxic to nematodes, we hypothesized that the eukaryotic cellular pathways that are affected by PCN could be evolutionarily conserved and, therefore, by using a plant model system, it would be possible to define whether PCN causes toxicity to cells or regulates fundamental cellular processes such as division, elongation, or differentiation at concentrations naturally present when colonizing host cells. Moreover, diverse bacterial species proliferate in the rhizosphere and release PCN and other phenazines with potential biocontrol activities (BosgelmezTinaz 2003; Fuqua and Greenberg 2002). This would suggest that natural phenazines such as phenazine-1-carboxylic acid and PCN can accumulate in the plant rhizosphere in amounts sufficient not only for inter- and intraspecies signaling but also for the direct inhibition of competing organisms.

Despite numerous reports of PCN-mediated cellular injuries, the response of plant cells or whole organs to $P$. aeruginosaproduced PCN is unknown. A lack of information might have led to an underestimation or miss-estimation of the mechanisms by which $P$. aeruginosa causes cell damage or phytostimulation. Knowledge about the activity of PCN in plants and the cellular pathways that are affected may be of practical value in agriculture, and it was the objective of this research to clarify some aspects of PCN activity in Arabidopsis seedlings. We found that PCN can be directly perceived by roots to adjust growth and development and no toxicity symptoms were evident, indicating that the activity of PCN in plants might be rather different from that reported for animal cells. PCN was found to inhibit primary root growth and stimulate lateral root and root hair formation in a dose-dependent way in low micromolar concentrations (Figs. 4 and 5). In this regard, PCN activity is similar to the previously reported activities of other bacterial QS signals (namely, C12-AHL), which regulate root system architecture in a highly specific way, depending on the length of the acyl-side chain (Ortiz-Castro et al. 2008). These results indicate that bacteria may affect root development by producing not only AHL but also PCN and, possibly, other phenazines with signaling roles in plant cells.

Accumulating evidence indicates that ROS play an essential role in the basic mechanism of cell growth and in the establishment of cell shape. This fundamental role in cell growth is likely to be widespread in plant parts, as shown in the polarized tip growth of root hairs. These structures are long, thin extensions growing out perpendicularly from trichoblasts, one of the cell types of the root epidermis. The presence of root hairs greatly increases the surface area of the root available for the absorption of nutrients and water and for interaction with soil particles and bacteria. Because PCN decreases trichoblast cell length and increases root hair elongation (Fig. 5), it is tempting to speculate that plant perception of QS signals and PCN affect both the production and localization of ROS and then the growth mechanisms that determine the shape of trichoblast change. Our data indicating that PCN affects primary roots, root hairs, and lateral root development through production of ROS are consistent with available genetic and pharmacological evidences. For instance, the ROOT HAIR DEFECTIVE2 (RHD2)/AtrbohC protein defective on a respiratory burst oxidase homolog $(\mathrm{RBOH})$ enzyme, which catalyzes the reduction of oxygen to generate the $\mathrm{O}^{2-}$ anion, is required for root hair elongation. The roots of plants homozygous for loss-of-function rhd2 mutations have decreased levels of ROS and are $20 \%$ shorter than the WT, indicating that cell expansion is defective in these plants (Foreman et al. 2003; Renew et al. 2005). On the other hand, by using inhibitors such as diphenylene iodonium, it has been suggested that NOX-derived ROS control cell expansion in maize (Zea mays) roots (Liszkay et al. 2004). Recently, it was found that silencing PvRbohB in transgenic Phaseolus vulgaris roots had a negative impact on LRD. In this work, the downregulation of PvRbohB affected both the growth and ROS levels in young lateral roots. Interestingly, the PvRbohB promoter was induced during lateral root primordium initiation in the pericycle, and remained active throughout lateral root development. This study identifies RBOHs as potentially important players in lateral root development in $P$. vulgaris. The particular impact of such regulation of root hair and lateral root growth by bacterial molecules such as PCN in the interactions between plants and bacteria remains to be determined. The above-described information indicates that ROS-mediated configuration of the root system is not an Arabidopsis-specific response and, thus, PCN might be active in crops.

Although root treatment with PCN did not induce visible cell death in transgenic Arabidopsis seedlings expressing the AtHisH2B:YFP marker stained with PI, a marked reduction in root meristem length and expression of $C y c B 1: u i d A$ and AtPRZ1:uidA was observed (Fig. 6), indicating that PCN repress cell division. It could be proposed that the PCN-induced generation of ROS might lower proliferating cell activity, thus decreasing primary root growth. These results suggest that redox regulation plays an important role in maintaining root meristem activity. Moreover, this is supported by previous findings that differences in $\mathrm{O}^{2-}$ and $\mathrm{H}_{2} \mathrm{O}_{2}$ accumulation in the root tip significantly affect root growth and differentiation (Dunand et al. 2007; Tsukagoshi et al. 2010). Our data indicate that PCN modulates the balance between cell proliferation and differentiation by directly regulating the accumulation of ROS in the root tip.

Contradictory information exists regarding the role of plant hormones in regulation of ROS production. Transcriptional regulation of ROS controls transition from proliferation to differentiation in the Arabidopsis primary root. This pathway seems to function independently of auxin and cytokinin signaling (Tsukagoshi et al. 2010). In contrast, in Lepidium sativum (cress), five respiratory burst oxidase homologs (Lesarbohs) were sequenced and it was found that their expression patterns were similar to their Arabidopsis orthologs throughout the life cycle. Cress plants in which LesarbohB expression was knocked down showed a root phenotype associated with defective auxin-related genes (Müller et al. 2012). These transgenic plants further displayed altered expression of auxin marker genes, including those encoding the auxin-responsive proteins 14 and 5 (IAA14 and IAA5), and LATERAL ORGAN BOUNDARIES DOMAIN16, an auxin-responsive protein implicated in lateral root initiation. It was speculated that ROS produced by rbohs play a role in root development via auxin signaling. Our data are in agreement with those of Tsukagoshi and associates (2010) in that the effects of PCN on ROS induction are independent of auxin signaling, considering the following evidence. First, PCN did not activate the expression of the auxin-inducible reporter markers DR5:uidA and BA3:uidA (Fig. 7); second, the auxin-related tirlafb2afb3, arf7 arf19, axr 1-3, and aux 1-7 mutants displayed similar primary root growth inhibition in response to PCN when compared with WT seedlings. In addition, the primary root growth of cytokinin- and abscisic acid-related mutants also were normally inhibited by PCN, indicating that the genes defective in these mutants are unlikely mediating the cellular effects of PCN in the primary root.

The ROS distribution at the primary root tip shows that localized $\mathrm{O}^{2-}$ accumulation in the meristematic zone is necessary for proliferation, whereas $\mathrm{H}_{2} \mathrm{O}_{2}$ accumulates in the elongation zone when cells arrest division and begin differentiation (Tsukagoshi et al. 2010). Because PCN is a redox-active compound and has been demonstrated before to be capable of generating ROS in animal systems, we investigated whether PCN 
treatment activates the oxidative machinery of Arabidopsis roots. By means of a combination of fluorophores that specifically react with ROS and using confocal microscopy, we found that PCN supply to Arabidopsis seedlings grown in vitro leads to enhanced $\mathrm{ROS}$ and $\mathrm{O}^{2-}$ levels in primary root tips. $\mathrm{PCN}$ treatment increased $\mathrm{O}^{2-}$ accumulation in the root elongation zone (Fig. 9), while co-cultivation with Pseudomonas aeruginosa (Fig. 2) or PCN supply (Fig. 10) decreased $\mathrm{H}_{2} \mathrm{O}_{2}$ accumulation in the same region, which was coincident with the inhibitory effects of PCN on cell division and elongation. Thus, disrupting the spatial distribution of $\mathrm{O}^{2-}$ or $\mathrm{H}_{2} \mathrm{O}_{2}$ may compromise normal root growth. Staining for the presence of these ROS in the root showed a clear correlation between growth rate and the relative distribution of different ROS species in the meristematic and elongation zones. Interestingly, differences in the localization of $\mathrm{O}^{2-}$ and $\mathrm{H}_{2} \mathrm{O}_{2}$ in seedlings treated with PCN (Fig. 9) or co-cultivated with P. aeruginosa WT and QS-related mutants lasI, rhlI, and rhlI/lasI (Fig. 2) suggest that these ROS can function as intercellular signaling molecules and not only as toxicity factors, as reported in animal cells.

The PCN-elicited accumulation of ROS was partially blocked when supplied together with the ethylene blocker $\mathrm{AgNO}_{3}$ (Fig. 9), and the $\mathrm{H}_{2} \mathrm{O}_{2}$ decrease was lower in the ethylene-related mutants etr $1-1$, ein2-1, and ein3-1 than in WT seedlings. These data correlate with the greater resistance of ethylene-related mutants etrl and ein2-1 to primary root growth inhibition caused by PCN (Fig. 8), further indicating that ethylene plays an important role in mediating the ROS response to PCN. To the best of our knowledge, the particular distribution of $\mathrm{O}^{2-}$ and $\mathrm{H}_{2} \mathrm{O}_{2}$ in primary root tips of ethylenerelated mutants has not been previously investigated. However, while analyzing the flg22-triggered ROS production in Arabidopsis seedlings, Mersmann and associates (2010) identified ethylene signaling as a critical component of the oxidative burst in response to this bacterial elicitor because etrl-1 and ein2-1 mutants were strongly diminished in flg22-induced ROS accumulation. Ethylene has diverse functions in plantmicrobe interactions (van Loon et al. 2006). It is important for defense against necrotrophic fungi (Chagué et al. 2006) but its contribution to bacterial resistance remains unclear. Our data demonstrated that, among the PCN responses tested, the ethylene-insensitive mutants were resistant to the PCN effect, decreasing $\mathrm{H}_{2} \mathrm{O}_{2}$ accumulation in the elongation zone of the primary root (Fig. 10). This suggests that ethylene plays a dual function in response to bacterially produced PCN: it may contribute to defense responses, possibly through regulation of ROS production, and, at the same time, in ROS-modulated root system architecture. Our work underscores the importance of PCN as a signaling molecule in plant-bacteria interactions as a modulator of cellular programs that determine the configuration of the root system. Understanding the contribution of QS in pathogenesis and symbiosis, particularly the role played by AHL in the production of virulence factors or compounds with a role in auxin (Ortiz-Castro et al. 2011) or ethylene signaling (this work), should contribute to the development of new strategies for protecting plants against pathogens or increase plant productivity.

\section{MATERIALS AND METHODS}

\section{Plant material and growth conditions.}

A. thaliana (Col-0); the transgenic lines CycB1:uidA (ColónCarmona et al. 1999), AtPRZ1:uidA (Sieberer et al. 2003), DR5: uidA (Ulmasov et al. 1997), BA3:uidA (Oono et al. 1998), and histone AtHisH2B:YFP (Boisnard-Lorig et al. 2001); and mutant lines etrl-1 (Hua and Meyerowitz 1998), ein2-1 (Guzmán and Ecker 1990), ein3-1 (Chao et al. 1997), tirlafb2afb3 (Dharmasiri et al. 2005), arf7arf19 (Okushima et al. 2007), auxl-7 (Pickett et al. 1990), axrl-3 (Lincoln et al. 1990), abil (Ma et al. 2009), abi3 (Koornneef et al. 1984; Nambara et al. 1992), and cre1-12ahk2-2 and cre1-12ahk3-3 (Higuchi et al. 2004; Mähönen et al. 2006) were used for all experiments. Seed were surface sterilized with $95 \%$ (vol/vol) ethanol for 5 min and $20 \%$ ( $\mathrm{vol} / \mathrm{vol}$ ) bleach for $7 \mathrm{~min}$. After five washes with sterile distilled water, seed were germinated and grown on agar plates containing $0.2 \times$ MS medium (Murashige and Skoog 1962). MS medium (Murashige and Skoog basal salts mixture; catalog M5524) was purchased from Sigma-Aldrich (St. Louis). The suggested formulation is salts at $4.3 \mathrm{~g} \mathrm{liter}^{-1}$ for a $1 \times$ concentration of medium; we used $0.9 \mathrm{~g} \mathrm{liter}^{-1}$, which we consider and refer to as $0.2 \times \mathrm{MS}$. This medium lacks amino acids and vitamins. PCN was purchased from Sigma-Aldrich. The compound was dissolved in dimethyl sulfoxide and used at the indicated concentrations. In control seedlings, we added the solvent in amounts equal to those present in the greatest concentration of compound tested. Phytagar (micropropagation grade) was purchased from Phytotechnology (Shawnee Mission, KS, US.A.). Plants were placed in a plant growth chamber (Percival Scientific AR-95L) with a photoperiod of $16 \mathrm{~h}$ of light, $8 \mathrm{~h}$ of darkness, light intensity of $100 \mu \mathrm{mol} \mathrm{m} \mathrm{m}^{2} \mathrm{~s}^{-1}$, and temperature of $22^{\circ} \mathrm{C}$.

\section{In vitro plant-bacteria co-cultivation assays.}

Bacterial strains used in this work were $P$. aeruginosa PAO1 (WT) and $P$. aeruginosa single mutants rhlI- and lasI- and double mutant rhlI-/lasI- (Li et al. 2007). The bacterial strains were evaluated in vitro for their pathogenic or plant-growthpromotion ability, using the Arabidopsis Col-0 ecotype. Bacterial densities of $2.5 \times 10^{8} \mathrm{CFU}$ were inoculated by streaking on agar plates containing $0.2 \times$ MS medium. Six-day-old germinated Arabidopsis seedlings (10 seedlings per plate) were transferred and located over the bacterial streak site and grown for a further 3-, 6-, and 9-day period. The plates were placed in the growth chamber (Percival Scientific AR-95L) in a completely randomized design. All experiments were replicated at least three times.

\section{Analysis of plant growth and statistical analysis.}

Growth of primary roots was registered using a rule. Lateral root number (LRN) was determined by counting the lateral roots present in the primary root from the tip to root/stem transition. LRD was determined by dividing the LRN by the primary root length and was expressed as LRD $\mathrm{cm}^{-1}$. The length of the meristems was determined as the distance between the quiescent centers to the cell file where cells started to elongate. For all experiments, data were statistically analyzed in the SPSS 10 program (SPSS, Chicago). Univariate and multivariate analyses with a Tukey's post hoc test were used for testing differences in growth and root developmental responses in the WT and ethylene-related mutants. Different letters are used to indicate means that differ significantly $(P<0.05)$.

\section{Microscopy.}

The $A$. thaliana root system was analyzed with a stereoscopic microscope (Leica MZ6; Leica Microsystems, Wetzlar, Germany). Total lateral roots were counted at $\times 30$ magnification. Primary root meristems were analyzed in semipermanent preparations of cleared roots using a composed microscope (Axiostar Zeiss Plus; Carl Zeiss, Göttingen, Germany) at $\times 100$ or $\times 400$ magnifications. Images were captured with a Sony Cybershot DSC-S75 digital camera (Sony Electonics Inc., Oradell, NJ, U.S.A.) adapted to the microscope and processed with the Zeiss Axio Vision 4AC software (Carl Zeiss). 
Histochemical analysis.

Transgenic plants that express the uidA reporter gene (Jefferson et al. 1987) were stained in 0.1\% 5-bromo-4-chlorium-3-indolyl, $\beta$-D-glucuronide in phosphate buffer $\left(\mathrm{NaH}_{2} \mathrm{PO}_{4}\right.$ and $\mathrm{Na}_{2} \mathrm{HPO}_{4}, 0.1 \mathrm{M}, \mathrm{pH}$ 7) with $2 \mathrm{mM}$ potassium ferrocyanide and $2 \mathrm{mM}$ potassium ferricyanide for $12 \mathrm{~h}$ at $37^{\circ} \mathrm{C}$. Plants were cleared and fixed as previously described by Malamy and Benfey (1997). The processed roots were included in glass slips and sealed with commercial nail varnish. For each marker line and treatment, at least 10 transgenic plants were analyzed.

$\mathrm{H}_{2} \mathrm{O}_{2}$ production was detected by the endogenous peroxidase-dependent staining procedure using 3,3'-diaminobenzidine (DAB) uptake (Thordal-Christensen et al. 1997). Control or PCN-treated of $A$. thaliana WT and ethylene mutant seedlings were placed in a solution of DAB at $1 \mathrm{mg} \mathrm{ml}^{-1}$, $\mathrm{pH} 3.8$, and incubated in dark for $2 \mathrm{~h}$. Subsequently, they were immersed in boiling $96 \%$ (vol/vol) ethanol for $10 \mathrm{~min}$ and then stored in $96 \%$ ( vol $/ \mathrm{vol})$ ethanol. For each treatment, at least 15 treated seedlings were analyzed. A representative plant was chosen for each treatment. $\mathrm{H}_{2} \mathrm{O}_{2}$ production was visualized as a reddish-brown precipitated coloration and photographed using a stereoscopic microscope.

\section{PI staining and YFP detection.}

For confocal microscopy, solvent- or PCN-treated transgenic Arabidopsis seedlings expressing the histone AtHisH2B:YFP construct (Boisnard-Lorig et al. 2001) were mounted on microscope slides into a solution of PI. For fluorescent staining with PI, recently collected plants with intact root systems were transferred to a solution of PI at $10 \mathrm{mg} / \mathrm{ml}$ for $3 \mathrm{~min}$. Seedlings were rinsed in water and mounted in $50 \%$ glycerol on microscope slides. The same sample was recorded separately at wavelengths specific to both PI fluorescence with an $568 \mathrm{~nm}$ excitation line and a emission window of 585to $610 \mathrm{~nm}$, and YFP emission with a 505- to 550-nm band-pass emission filter (488-nm excitation line), after which the two images were merged to produce the final image. Primary root meristems were analyzed by imaging mounted samples with an inverted confocal microscope (Olympus FV1000).

\section{PCN quantification.}

PCN was extracted from the supernantant fraction of $P$. aeruginosa grown in Luria-Bertani medium at $37^{\circ} \mathrm{C}$ for $48 \mathrm{~h}$. Supernatant $(1 \mathrm{ml})$ was mixed with $1 \mathrm{ml}$ of chloroform and the lower organic layer was separated. To this layer, $1 \mathrm{ml}$ of $0.2 \mathrm{HCl}$ was added and the PCN rich organic layer was separated to give a pink to deep-red solution. The absorbance of this solution was measured at $520 \mathrm{~nm}$. Concentrations, expressed as micrograms of PCN produced per milliliter of culture supernantant, were determined according to Essar and associates (1990).

\section{ROS and $\mathrm{O}^{2-}$ detection}

General ROS and specific $\mathrm{O}^{2-}$ anion were monitored by incubating Arabidopsis seedlings with $10 \mu \mathrm{M}$ fluorescent probes H2DCF-DA and DHE, respectively, in $10 \mathrm{mM}$ Tris$\mathrm{HCl}$ (pH 7.4) (Gomes et al. 2005). Arabidopsis-treated seedlings were incubated for $30 \mathrm{~min}$ in darkness and washed three times for 5 min with fresh buffer. Fluorescence signals from at least 10 treated and control seedlings were detected using a confocal microscope (Olympus FV1000). Fluorescence signals were quantified by counting pixel numbers in the green channel by employing ImageJ software.

\section{ACKNOWLEDGMENTS}

This work was supported by grants from the Consejo Nacional de Ciencia y Tecnología (CONACYT, México, grant numbers 60999 and 177775), the Consejo de la Investigación Científica (UMSNH, México, grant number CIC 2.26), and the Marcos Moshinsky Fellowship to J. López-Bucio. We thank P. Doerner, C. Luschnig, T. Guilfoyle, and P. Guzman for kindly providing us with seed of transgenic and mutant lines and A. Méndez Bravo for his kind support with confocal microscopy analysis.

\section{LITERATURE CITED}

Boisnard-Lorig, C., Colon-Carmona, A., Bauch, M., Hodge, S., Doerner P., Bancharel, E., Dumas, C., Haseloff, J., and Berger, F. 2001. Dynamic analyses of the expression of the HISTONE::YFP fusion protein in Arabidopsis show that syncytial endosperm is divided in mitotic domains. Plant Cell 13:495-509.

Bosgelmez-Tinaz, G. 2003. Quorum sensing in gram-negative bacteria. Turk. J. Biol. 27:85-93.

Chagué, V., Danit, L. V., Siewers, V., Schulze-Gronover, C., Tudzynski, P., Tudzynski, B., and Sharon, A. 2006. Ethylene sensing and gene activation in Botrytis cinerea: A missing link in ethylene regulation of fungus-plant interactions. Mol. Plant-Microbe Interact. 19:33-42.

Chao, Q., Rothenberg, M., Solano, R., Roman, G., Terzaghi, W., and Ecker, J. R. 1997. Activation of the ethylene gas response pathway in Arabidopsis by the nuclear protein ETHYLENE-INSENSITIVE3 and related proteins. Cell 27:1133-1144.

Colón-Carmona, A., You, R., Haimovitch-Gal, T., and Doerner, P. 1999. Spatio-temporal analysis of mitotic activity with a labile cyclin-GUS fusion protein. Plant J. 20:503-508.

De Kievit, T. R., and Iglewski, B.H. 2000. Bacterial quorum sensing in pathogenic relationships. Infect. Immun. 68:4839-4849.

De Vleesschauwer, D., Cornelis, P., and Höfte, M. 2006. Redox-active pyocyanin secreted by Pseudomonas aeruginosa 7NSK2 triggers systemic resistance to Magnaporthe grisea but enhances Rhizoctonia solani susceptibility in rice. Mol. Plant-Microbe Interact. 19:14061419.

Dharmasiri, N., Dharmasiri, S., and Estelle, M. 2005. The F-box protein TIR1 is an auxin receptor. Nature 435:441-445.

Dietrich, L. E. P., Price-Whelan, A., Petersen, A., Whiteley, M., and Newman, D. K. 2006. The phenazine pyocyanin is a terminal signaling factor in the quorum sensing network of Pseudomonas aeruginosa. Mol. Microbiol. 61:1308-1321.

Dunand, C., Crevecoeur, M., and Penel, C. 2007. Distribution of superoxide and hydrogen peroxide in Arabidopsis root and their influence on root development: Possible interaction with peroxidases. New Phytol. 174:332-341.

Essar, D. W., Eberly, L., Hadero, A., Crawford, I. P. 1990. Identification and characterization of genes for a second anthranilate synthase in Pseudomonas aeruginosa: Interchangeability of the two anthranilate synthases and evolutionary implications. J. Bacteriol. 172:884-900.

Foreman, J., Demidchick, V., and Bothwell, J. H. F. 2003. Reactive oxygen species produced by NADPH oxidase regulate plant cell growth. Nature 422:442-446.

Friesen, M. L., Porter, S. S., Stark, S. C., von Wettberg, E. J., Sachs, J. L., and Martinez-Romero, E. 2011. Microbially mediated plant functional traits. Annu. Rev. Ecol. Evol. Syst. 42:23-46.

Fuqua, C., and Greenberg, E. P. 2002. Listening in on bacteria: Acylhomoserine lactone signalling. Nat. Rev. Mol. Cell Biol. 3:685-695.

Fuqua, W. C., Winans, S. C., and Greenberg, E. P. 1994. Quorum-sensing in bacteria: The luxR-LuxI family of cell density-responsive transcriptional regulators. J. Bacteriol. 176:269-275.

Gomes, A., Fernandes, E., and Lima, J. L. F. C. 2005. Fluorescence probes used for detection of reactive oxygen species. J. Biochem. Biophys. Methods 65:45-80.

Guzmán, P., and Ecker, J. R. 1990. Exploiting the triple response of Arabidopsis to identify ethylene-related mutants. Plant Cell 2:513-523.

Higuchi, M., Pischke, M. S., Mahonen, A. P., Miyawaki, K., Hashimoto, Y., Seki, M., Kobayashi, M., Shinozaki, K., Kato, T., Tabata, S., Heleriutta, Y., Sussman, M. R., and Kakimoto, T. 2004. In planta functions of the Arabidopsis cytokinin receptor family. Proc. Natl. Acad. Sci. U.S.A. 101:8821-8826.

Hua, J., and Meyerowitz, E. 1998. Ethylene responses are negatively regulated by a receptor gene family in Arabidopsis thaliana. Cell 94:262271.

Hunter, R. C., Klepa-Ceraj, V., Lorenzi, M .M. Grotzinger, H. Martin, T. R., and Newman, D. K. 2012. Phenazine content in the cystic fibrosis respiratory tract negatively correlates with lung function and microbial complexity. Am. J. Respir. Cell Mol. Biol. 47:738-745.

Jefferson, R. A., Kavanagh, T. A., and Bevan, M. W. 1987. GUS fusion: $\beta$ glucuronidase as a sensitive and versatile fusion marker in higher plants. EMBO (Eur. Mol. Biol. Organ.) J. 6:3901-3907.

Koornneef, M., Reuling, G., and Karssen, C. M. 1984. The isolation and 
characterization of abscisic acid-insensitive mutants of Arabidopsis thaliana. Physiol. Plant. 61:377-383.

Lau, G. W., Goumnerov, B. C., Walendziewicz, C. L., Hewitson, J., Xiao, W., Mahajan-Miklos, S., Tompkins, R. G., Perkins, L. A., and Rahme, L. G. 2003. The Drosophila melanogaster toll pathway participates in resistance to infection by the gram-negative human pathogen Pseudomonas aeruginosa. Infect. Immun. 71:4059-4066.

Lau, G. W., Hassett, D. J., Ran, H., and Kung, F. 2004a. The role of pyocyanin in Pseudomonas aeruginosa infection. Trends Mol. Med. 10:599606.

Lau, G. W., Ran, H., Kong, F., Hassett, D. J., and Mavrodi, D. 2004b. Pseudomonas aeruginosa pyocyanin is critical for lung infection in mice. Infect. Immun. 72:4275-4278.

Li, L. L., Malone, J. E., and Iglewski, B. H. 2007. Regulation of the Pseudomonas aeruginosa quorum-sensing regulator VqsR. J. Bacteriol. 189:4367-4374.

Lincoln, C., Britton, J. H., and Estelle, M. 1990. Growth and development of the axr1 mutant of Arabidopsis. Plant Cell 2:1071-1080.

Liszkay, A., van der Zalm, E., and Schopfer, P. 2004. Production of reactive oxygen intermediates by maize roots and their role in wall loosening and elongation growth. Plant Physiol. 136:3114-3123.

Liu, G. Y., and Nizet, V. 2009. Color me bad: Microbial pigments as virulence factors. Trends Microbiol. 17:406-413.

Ma, Y., Szostkiewicz, I., Korte, A., Moes, D., Yang, Y., Christmann, A., and Grill, E. 2009. Regulators of PP2C phosphate activity function as abscisic acid sensors. Science 324:1064-1068.

Mahajan-Miklos, S., Tan M. W., Rahme, L. G., and Ausubel, F. M. 1999. Molecular mechanisms of bacterial virulence elucidated using a Pseudomonas aeruginosa-Caenorhabditis elegans pathogenesis model. Cell 96:47-56.

Mähönen, A. P., Bishopp, A., Higuchi, M., Nieminen, K. M., Kinoshita, K., Törmäkangas, K., Ikeda, Y., Oka, A., Kakimoto, T., and Helariutta, Y. 2006. Cytokinin signaling and its inhibitor AHP6 regulate cell fate during vascular development. Science 311:94-98.

Malamy, J. E., and Benfey, P. N. 1997. Organization and cell differentiation in lateral roots of Arabidopsis thaliana. Development 124:33-44.

Mathesius, U., Mulders, S., Gao, M., Teplitski, M., Caetano-Anollés, G., Rolfe, B. G., and Bauer, W. 2003. Extensive and specific responses of a eukaryote to bacterial quorum-sensing signals. Proc. Natl. Acad. Sci. U.S.A. 100:1444-1449.

Mavrodi, D. V., Bunsall, R. F., Delaney, S. M., Soule, M. J., Phillips, G., and Thomashow, L. S. 2001. Functional analysis of genes for biosynthesis of pyocyanin and phenazine-1-carboxamide from Pseudomonas aeruginosa PAO1. J. Bacteriol. 183:6454-6465.

Mersmann, S., Bourdais, G., Rietz, S., and Robatzek, S. 2010. Ethylene signaling regulates accumulation of the FLS2 receptor and is required for the oxidative burst contributing to plant immunity. Plant Physiol 154:391-400.

Miller, M. B., and Bassler, B. L. 2001. Quorum sensing in bacteria. Annu. Rev. Microbiol. 55:165-199.

Müller, K., Linkies, A., Leubner-Metzger, G., and Kermode, A. R. 2012. Role of a respiratory burst oxidase of Lepidium sativum (cress) seedlings in root development and auxin signalling. J. Exp. Bot. 63:63256334.

Murashige, T., and Skoog, F. 1962. A revised medium for rapid growth and bioassays with tobacco tissue culture. Physiol. Plant. 15:473-497.

Nambara, E., Naito, S., and McCourt, P. 1992. A mutant of Arabidopsis which is defective in seed development and storage protein accumulation is a new abi3 allele. Plant J. 2:435-441.

Okushima, Y., Fukaki, H., Onoda, M., Theologis, A., and Tasaka, M. 2007. ARF7 and ARF19 regulate lateral root formation via direct activation of LBD/ASL genes in Arabidopsis. Plant Cell 19:118-130.

O’Malley, Y. Q., Reszka, K. J., Spitz, D. R., Denning, G. M., and Britigan, B. E. 2004. Pseudomonas aeruginosa pyocyanin directly oxides glutathione and decreases its levels in airway epithelial cells. Am. J. Physiol. Lung Cell Mol. Physiol. 287:94-103.

Oono, Y., Chen, Q. G., Overvoorde, P. J., Kohler, C., and Theologis, A. 1998. age mutants of Arabidopsis exhibit altered auxin-regulated gene expression. Plant Cell 10:1649-1662.

Ortiz-Castro, R., and López-Bucio, J. 2013. Small molecules involved in transkingdom communication between plants and rhizobacteria. Pages 295-307 in: Molecular Microbial Ecology of the Rhizosphere, Vol. I. F.
J. De Bruijn, ed. Wiley-Blackwell, Hoboken, NJ, U.S.A.

Ortiz-Castro, R., Martínez-Trujillo, M., and López-Bucio, J. 2008. N-acylL-homoserine lactones: A class of bacterial quorum-sensing signals alter post-embryonic root development in Arabidopsis thaliana. Plant Cell Environ. 31:1497-1509.

Ortiz-Castro, R., Contreras-Cornejo, H. A., Macías-Rodriguez, L., and López-Bucio, J. 2009. The role of microbial signals in plant growth and development. Plant Signal. Behav. 4:1-12.

Ortiz-Castro, R., Díaz-Pérez, C., Martínez-Trujillo, M., del Río, R. E., Campos-García, J., and López-Bucio, J. 2011. Transkingdom signaling based on bacterial cyclodipeptides with auxin activity in plants. Proc. Acad. Natl. Sci. U.S.A. 108:7253-7258.

Pickett, F. B., Wilson, A. K., and Estelle, M. 1990. The auxl mutation of Arabidopsis confers both auxin and ethylene resistance. Plant Physiol. 94:1462-1466.

Price-Whelan, A., Dietrich L. E. P., and Newman, D. K. 2007. Pyocyanin alters redox homeostasis and carbon flux through central metabolic pathways in Pseudomonas aeruginosa PA14. J. Bacteriol. 189:63726381

Rada, B., and Leto, T. 2013. Pyocyanin effects on respiratory epithelium: Relevance in Pseudomonas aeruginosa airway infections. Trends Microbiol. 21:73-81.

Renew, S., Heyno, E., Schopfer, P., and Liszkay, A. 2005. Sensitive detection and localization of hydroxyl radical production in cucumber roots and Arabidopsis seedlings by spin trapping electron paramagnetic resonance spectroscopy. Plant J. 44:342-347.

Rumbaugh, K. P., Griswold, J. A., Iglewski, B. H., and Hamood, A. N. 2000. The role of quorum sensing in the in vivo virulence of Pseudomonas aeruginosa. Microbes Infect. 2:1721-1731.

Schaber, J. A., Carty, N. L., McDonald, N. A., Graham, E. D., Cheluvappa, R., Griswold, J. A., and Hamood, A. N. 2004. Analysis of quorum sensing-deficient clinical isolates of Pseudomonas aeruginosa. J. Med. Microbiol. 53:841-853.

Sieberer, T., Hauser, M. T., Seifert, G. J., and Lusching, C. 2003. PROPORZ1, a putative Arabidopsis transcriptional adaptor protein, mediates auxin and cytokinin signals in the control of cell proliferation. Curr. Biol. 13:837-842.

Siehnel, R., Traxler, B., An, D. D., Parsek, M. R., Schaefer, A. L., and Singh, P. K. 2010. A unique regulator controls the activation threshold of quorum-regulated genes in Pseudomonas aeruginosa. Proc. Natl. Acad. Sci. U.S.A. 107:7916-7921.

Thordal-Christensen, H., Zhang, Z., Wei, Y., and Collinge, D. B. 1997. Subcellular localization of $\mathrm{H}_{2} \mathrm{O}_{2}$ in plants. $\mathrm{H}_{2} \mathrm{O}_{2}$ accumulation in papillae and hypersensitive response during the barley-powdery mildew interaction. Plant J. 11:1187-1194.

Tsukagoshi, H., Busch, W., and Benfey, P. B. 2010. Transcriptional regulation of ROS controls transition from proliferation to differentiation in the root. Cell 143:606-615.

Ulmasov, T., Murfett, J., Hagen, G., and Guilfoyle, T. 1997. Aux/IAA proteins repress expression of reporter genes containing natural and highly active synthetic auxin response elements. Plant Cell 9:1963-1971.

van Loon, L. C., Geraats, B. P. J., and Linthorst, H. J. M. 2006. Ethylene as a modulator of disease resistance in plants. Trends Plant Sci. 11:184 191.

Venturi, V. 2006. Regulation of quorum sensing in Pseudomonas. FEMS (Fed. Eur. Microbiol. Soc.) Microbiol. Rev. 30:274-291.

Verhagen, B. W. M., Trotel, P., Couderchet, M., Höfte, M., and Azziz, A. 2010. Pseudomonas spp.-induced systemic resistance to Botrytis cinerea is associated with induction and priming of defence responses in grapevine. J. Exp. Bot. 61:249-260.

von Rad, U., Klein, I., Dobrev, P. I., Kottova, J., Zazimolova, E., Fekete, A., Hartmann, A., Schmitt-Kopplin, P., and Durner, J. 2008. Response of Arabidopsis thaliana to N-hexanoyl-DL-homoserine-lactone, a bacterial quorum sensing molecule produce in the rhizosphere. Planta 229:73-85.

Wilson, R., Sykes, D. A., Watson, D., Rutman, A., Taylor, G. W., and Cole, P. J. 1998. Measurement of Pseudomonas aeruginosa phenazine pigments in sputum and assessment of their contribution to sputum toxicity for respiratory epithelium. Infect. Immun. 56:2515-2517.

Zamioudis, C., Mastranesti, P., Dhonukshe, P., Blilou, I., and Pieterse, C. M. J. 2013. Unraveling root developmental programs initiated by beneficial Pseudomonas spp. bacteria. Plant Physiol. 162:304-318. 